W. Tian. X. Han, W. Zuo, M. Sohn 2018. "Building Energy Simulation Coupled with CFD for Indoor Environment: A Critical Review and Recent Applications." Energy and Buildings, 165, pp.184-199. DOI:

10.1016/j.enbuild.2018.01.046.

\title{
Building Energy Simulation Coupled with CFD for Indoor Environment: A Critical Review and Recent Applications
}

\author{
Wei $\operatorname{Tian}^{\mathrm{a}+}, \mathrm{Xu} \mathrm{Han}^{\mathrm{b}}$, Wangda Zuob ${ }^{\mathrm{b}}$, Michael D. Sohn ${ }^{\mathrm{c}}$ \\ ${ }^{a}$ Department of Civil, Architectural and Environmental Engineering, University of Miami, \\ 1251 Memorial Drive, Coral Gables, FL 33146, U.S.A. \\ ${ }^{b}$ Department of Civil, Environmental and Architectural Engineering, University of Colorado \\ Boulder, UCB 428, Boulder, CO 80309, U.S.A. \\ ${ }^{c}$ Energy Analysis and Environmental Impacts Division, Lawrence Berkeley National \\ Laboratory, 1 Cyclotron Road, Berkeley, CA 94720, U.S.A. \\ ${ }^{+}$Current Affiliation: Schneider Electric, 800 Federal Street, Andover, MA 01810, U.S.A.
}

\section{ABSTRACT}

This paper presents a comprehensive review of the open literature on motivations, methods and applications of linking stratified airflow simulation to building energy simulation (BES). First, we review the motivations for coupling prediction models for building energy and indoor environment. This review classified various exchanged data in different applications as interface data and state data, and found that choosing different data sets may lead to varying performance of stability, convergence, and speed for the co-simulation. Second, our review shows that an external coupling scheme is substantially more popular in implementations of co-simulation than an internal coupling scheme. The external coupling is shown to be generally faster in computational speed, as well as easier to implement, maintain and expand than the internal coupling. Third, the external coupling can be carried out in different data synchronization schemes, including static coupling and dynamic coupling. In comparison, the static coupling that performs data exchange only once is computationally faster and more stable than the dynamic coupling. However, concerning accuracy, the dynamic coupling that requires multiple times of data exchange is more accurate than the static coupling. Furthermore, the review identified that the implementation of the external coupling can be achieved through customized interfaces, middleware, and standard interfaces. The customized 
interface is straightforward but may be limited to a specific coupling application. The middleware is versatile and user-friendly but usually limited in data synchronization schemes. The standard interface is versatile and promising, but may be difficult to implement. Current applications of the co-simulation are mainly energy performance evaluation and control studies. Finally, we discussed the limitations of the current research and provided an overview for future research.

Key words: Coupled Simulation, Building Energy Simulation, Computational Fluid Dynamics

\section{Introduction}

Building energy simulation (BES) is used to predict the building energy performance (Crawley et al. 2008; Harish and Kumar 2016). To model the indoor airflow in buildings, a lot of popular BES programs, such as EnergyPlus (Crawley et al. 2001), TRNSYS (Trnsys 2000), ESP-r (Strachan et al. 2008), IDA-ICE (IDA-ICE 2014), BSIM (Wittchen et al. 2005), etc., use a multizone modelling approach for its reasonable computation time and easy implementation (Foucquier et al. 2013). The multizone model (Axley 2007) considers each building zone as a node with homogeneous distribution of temperature, pressure, concentration, etc.

Recently, ventilation systems that involve stratified airflow distributions are emerging, as they can achieve better thermal comfort and energy performance. Examples are displacement ventilation, natural ventilation, and advanced ventilation methods for large spaces or spaces with high heat gain. However, adopting the multizone model that assumes that air is well mixed in a zone, BES may fail to simulate the stratified airflow with nonuniform temperature distributions (displacement ventilation) and strong momentum forces (pressure-driven natural ventilation) (Wang et al. 2012). In addition, the multizone model cannot predict the local thermal comfort distribution of the occupant zone, which can be used to control the Heating, Ventilation, and Air-Conditioning (HVAC) system (Crawley et al. 2008).

Different from the multizone model, computational fluid dynamics (CFD) decomposes a building zone into a large amount of control volumes and can provide detailed description of the airflow by solving the 
Navier-Stokes equations (Chen 2009). CFD has been successfully applied to predict detailed information of the airflow and the temperature distribution for various purposes (Liu et al. 2015; Zhai et al. 2002). Nevertheless, CFD has its own technical shortcomings. Besides a high demand in computational efforts, results of CFD are sensitive to the boundary conditions. However, current CFD programs usually do not have embedded, sophisticated, or rigorously-validated models to determine the dynamic boundary conditions in buildings. Thus, in standalone CFD simulations, users can study only a few design or operational scenarios by adopting fixed boundary conditions, such as fixed supply airflow rate and temperature, fixed wall temperature or heat flux through the envelopes, even though the actual boundary conditions are dynamically changing (Djunaedy et al. 2003).

To address the above-mentioned limitations, BES and CFD can be coupled to provide the critical information mutually, and achieve better results than standalone programs. On one hand, BES has validated HVAC system and thermal load prediction models that can provide dynamic boundary conditions to CFD. CFD, on the other hand, can provide the local airflow information to BES to study HVAC control and improve the thermal load calculation. Thus, the coupled simulation between BES and CFD can be utilized to seek a holistic solution for the design and operation of low-energy buildings (Tian and Zuo 2013).

This paper presents a thorough and critical review of recent advancement in the coupled simulation of building energy systems and the indoor environment. The paper is structured as follows: we first discuss the coupling method including the exchanged data, coupling schemes, data synchronization schemes, and implementation strategies. Then, we summarize the applications of the coupled simulation with a focus on studying energy and control performance of HVAC systems. Afterwards, we discuss the research gap and future trend of the coupled simulation. Eventually, we make concluding remarks on the coupled simulation between BES and CFD.

\section{Method}

\subsection{Exchanged Data}

The exchanged data between BES and CFD can be classified into two categories: interface data and 
state data. The interface data is defined as a set of data at the boundary of the two physical domains (HVAC system and indoor environment). The state data is defined as a set of data that belongs to either of the physical domains. Figure 1 shows a typical displacement ventilation system. Supposing that CFD is employed to simulate the airflow in the room while BES is to simulate the HVAC system, the interface data includes supply and return air temperature and velocity, and the state data includes the temperature at the thermostat location and Predicted Mean Vote/Predicted Percentage of Dissatisfied (PMV/PPD) for the occupant zone, which are used for control purposes of the HVAC. Note that the interface data is usually measurable while the state data is not necessarily to be measurable.

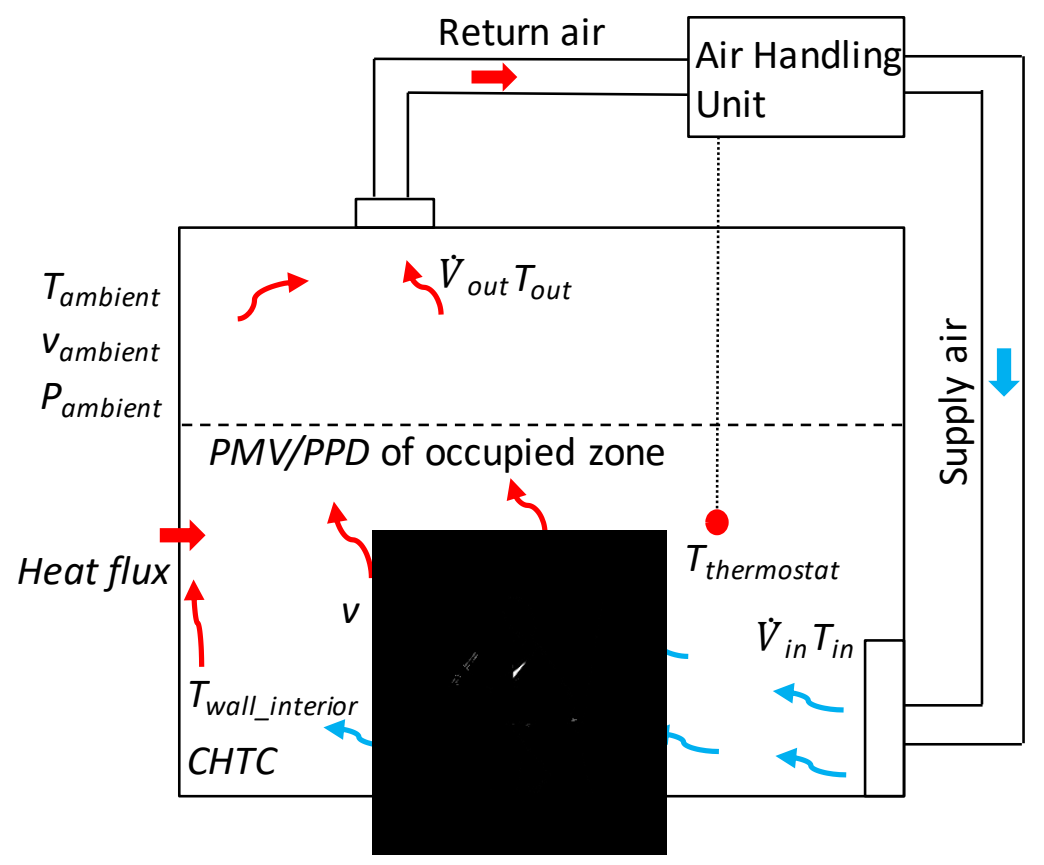

Figure 1 Exchanged data in a displacement ventilation system

The selection of the interface data and state data depends on the physical properties of the coupled domains and users' interpretation of them. For example, in the aforementioned case, the interface data can be velocity or total pressure, which is essentially determined by the fluid dynamics principles. To control the HVAC system, users determine the state data by choosing either the temperature at the thermostat or the PMV/PPD, which is completely subject to the interpretation by the users. In order to better guide the readers 
on the determination of the exchanged data, we conducted a thorough search on literature dealing with various co-simulation applications and summarized the possible exchanged data from BES to CFD including, but not limited to:

- Interface data: Interior surface temperature or heat flux of the envelopes. BES is coupled with CFD to improve the prediction accuracy of heat flux through the envelopes. In the first case, BES gives the interior surface temperature to CFD as boundary conditions and CFD then calculates the $\mathrm{CHTC}$ and heat flux through each wall by adding the heat flux between a solid wall and each adjacent fluid cell (Zhai et al. 2002; Zhang et al. 2012a). In the second case, BES gives heat flux to CFD as boundary conditions and CFD returns surface temperature back to BES. Novoselac (2004) pointed out that this can avoid inaccurate calculation of heat flux associated with wall functions in CFD.

- Interface data: Airflow rate (velocity, total pressure) and temperature at the openings (inlet, outlet, cracks, etc). The indoor airflow and HVAC systems are physically coupled through these openings. Thus, with the data at fluid openings provided by BES that simulates the HVAC system, CFD can then be used to predict the indoor environment (Fan and Ito 2012; Ascione et al. 2013; Zuo, Wetter, et al. 2016; Zuo et al. 2014).

- Interface data: Ambient airflow conditions. BES usually stores the annual weather data at various locations to simulate the whole year building energy performance. EnergyPlus, for example, provides a locally recorded data or a typical meteorological year weather data, which synthetically represents the outdoor weather condition (such as solar radiation, temperature, etc.) within a period of record (DOE 2017). With the temperature and total pressure data at the inlets of the building, CFD can predict the airflow and temperature distribution in the building and in turn help refine the design (Ohba and Lun 2010; Wang and Wong 2008).

Likewise, the possible exchanged data from CFD to BES include, but are not limited to:

- Interface data: Convective Heat Transfer Coefficient (CHTC). $\mathrm{CHTC}$ is critical to the calculation of heat flux through the envelopes. As reported in the research (Lomas 1996), the estimated annual 
heating energy based on four empirical formulas varies by about $27 \%$. As the value of CHTC is associated with the temperature stratification near the wall, the multizone model that assumes well-mixed air in BES cannot predict it satisfactorily. Thus, CFD is needed to determine the CHTC.

By simulating the detailed heat transfer phenomena near the wall, the CFD can provide an accurate and localized CHTC for the BES (Zhang et al. 2012b; Rong et al. 2011; Zhai et al. 2002).

- Interface data: $\underline{\text { Heat flux through the envelopes or temperature at the interior surface of the }}$ envelope. This is identical to the exchanged data from BES to CFD.

- Interface data: Airflow rates (total pressure, velocity) and temperature at openings in the buildings. The data at the openings between different air zones can be used in coupled simulation to study the airflow distribution within the whole buildings. Taking the building with atrium as an example, CFD is used to model the atrium due to the strong temperature stratification along the height, while BES is used to simulate the rest of rooms and spaces that are connected to the atrium. CFD calculates the total pressure or airflow rates at downstream (total pressure and temperature at upstream) and sends them back to the BES for airflow prediction using multizone models (Zhang et al. 2012a; Wang and Wong 2008; Pan et al. 2010).

- Interface data: Ambient airflow conditions at a small scale. As stated previously, BES stores the weather data based on the measurements at weather stations, which might be far away from the location of the simulated building. With the influence from other buildings and constructions on the path, the weather profile near the building can differ from the one stored in the BES. Thus, CFD can be adopted to quantify the influence and predict the weather profile in the microclimate, which can be used in BES to determine the performance of the natural ventilation (Wang 2007; Bouyer et al. 2011; Yi and Malkawi 2008).

- $\quad$ State data: PMV/PPD, temperature, humidity and speed. CFD has the capability to predict nonuniform local airflow and temperature distributions. This information can then be extracted and sent to BES for simulation of HVAC control (Tian, Sevilla, Zuo, et al. 2017; Tian et al. 2018; Liu et al. 2015; Ascione et al. 2013). 


\subsection{Coupling Mechanisms}

As shown in Figure 2, we divide the coupling mechanisms into two categories according to how the models are solved: internal coupling and external coupling. Internal coupling fuses one model into the other and has only one solver. External coupling links BES and CFD through an intermediate agent and keeps the integrity of each model. Details of the intermediate agent will be discussed in Section 2.4. Note that whether two programs are compiled together does not necessarily define the mechanism of the coupling. For example, one can program BES and CFD in one executable file. As long as any of them has a dedicated solver and exchanges data with the other, it is still regarded as external coupling. On the other hand, if coupling two models by using Functional Mock-up Interface (FMI) for Model Exchange and solving them only using importing model's solver, it is then regarded as internal coupling.

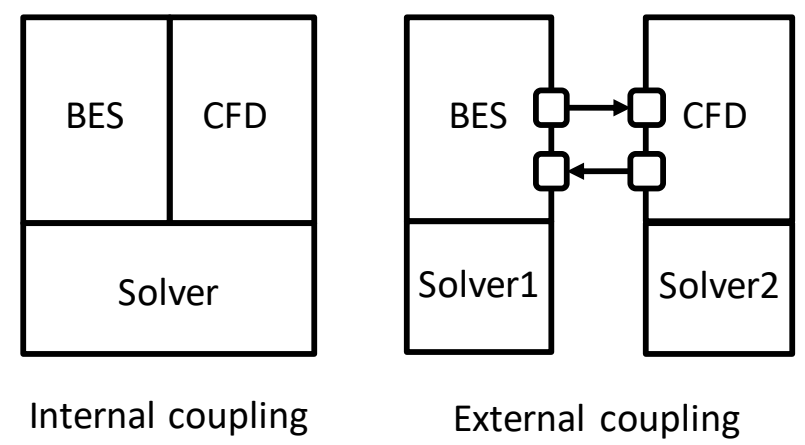

Figure 2 Schematic of internal coupling and external coupling

Internal coupling is theoretically accurate as it solves all the equations in a rigorous way. All the equations are supposed to be solved simultaneously. As mentioned before, the Functional Mock-up Interface (FMI) (Blochwitz, Otter, Arnold, Bausch, Elmqvist, et al. 2011) is receiving increasing attention and it can make the internal coupling much easier to implement. We will discuss FMI in Section 2.4.3. However, there are several major limitations (the first two of them may not be applied to internal coupling by FMI) in the internal coupling mechanism: 
- It can be costly in development and maintenance. Prior to the implementation of internal coupling, the developer should have the source codes of both programs (which means commercial and proprietary programs cannot be utilized). Delving into existing BES or CFD and localizing the part to fuse two programs can be challenging. Thus, researchers prefer to use their own BES or CFD program that is tailored to solve their problem (Chen et al. 1995). However, by using in-house codes, the users can hardly take advantage of the state-of-art BES and CFD programs, which are joint efforts between many institutions and huge in its project size, such as EnergyPlus (NREL 2017) and OpenFOAM (OpenFOAM Foundation 2017).

- It is costly in computation time (Zhai and Chen 2003). The physical domains in a building may have different time scales. For example, contaminant transport in indoor airflow models may have a time scale of less than one second, while thermal storage of the wall may have a time scale of a few hours. To solve them simultaneously in the internal coupling mechanism, a unified time step size (smaller time step size of the two domains in simulation) should be used. Thus, being strongly associated with the small time step size, the computational efforts can increase significantly.

- In addition to more demanding computational efforts, solving those equations simultaneously can impose additional challenges on the solver.

On the other hand, external coupling through linking two programs externally have a few advantages as follows:

- It is easier to be implemented (Trcka and Hensen 2006). The external coupling is not limited to open source programs, and it can also be applied to proprietary and commercial programs. Users just need to know the interface of each program instead of delving into the source codes. Moreover, without requiring to rewrite or modify the source codes, external coupling allows users to utilize the updated version of each program as long as the interface does not change.

- It might be less computational demanding and easier to harness parallel computing and distributed computing. First, two programs, being externally linked, run separately with their own time step size. For example, BES can use a time step in a scale of a minute to simulate heat transfer through 
the walls and CFD can use a time step size in a scale of a second to simulate airflow. The simulation speed of BES can be significantly improved, compared to that in internal coupling which normally requires a time step in a scale of second for both programs. Second, simulation speed can be further increased by taking full advantage of the computing power on the computer. For example, BES can run on central processing unit (CPU) while CFD can run in parallel on graphics processing unit (GPU) (Tian, Sevilla, and Zuo 2017).

\subsection{Data Synchronization Schemes in External Coupling}

As shown in Table 1, we can divide the schemes of data synchronization between CFD and BES into four categories based on the direction and number of data exchange. One-way data exchange indicates that only one program gives the data to the other while two-way data exchange indicates that data is exchanged between BES and CFD mutually. If the data exchange is executed only once for the entire simulation, it is called static synchronization. Otherwise, it is called dynamic synchronization. In the rest of the section, all data synchronization schemes will be discussed in detail. For ease of writing, we use simulator in the rest of the section to represent BES or CFD.

Table 1 List of data synchronization schemes

\begin{tabular}{|c|c|c|}
\hline & One-Way Data Exchange & Two-Way Data Exchange \\
\hline Static Synchronization & & Two-way static \\
\hline (one-time data exchange) & One-way Static & Quasi-dynamic \\
\hline $\begin{array}{l}\text { Dynamic Synchronization } \\
\text { (multiple data exchanges) }\end{array}$ & One-way Dynamic & Fully Dynamic \\
\hline
\end{tabular}

\subsubsection{Static Data Synchronization Scheme}

1) One-way Static Scheme 
In this scheme, only one simulator (either BES or CFD) sends data $x_{1}(0)$ at the beginning of the simulation to the other (Figure 3). The data exchange process is straightforward, so that it might be executed manually. Since there is only one data exchange, the overhead for data exchange is negligible. The procedure of the scheme is as follows:

Step 0: Initialize the simulator 2 and set time step size as $\Delta t_{2}$.

Step 1: Simulator 1 is called to generate the input data $x_{1}(0)$ for simulator 2.

Step 2: Simulator 2 generates an internal state after $j$ time step calculation as:

$$
x_{2}\left(t_{0}+j \Delta t_{2}\right)=f_{2}\left(x_{2}\left(t_{0}+(j-1) \Delta t_{2}\right), x_{1}(0)\right)
$$

Step 3: If $j=$ Last Step stop, else go to Step 2.

Note that we have the notations of data synchronization time points $t_{1}, \ldots, t_{n+1}$ in the figure to keep it consistent with the rest of data synchronization schemes. As a convention, we use $x_{2}(i)$ where $i \in \mathbb{N}$ to represent the exchanged data, and $x_{2}(t)$ where $t \in \mathbb{R}$ to represent the internal state of the simulator at time $t$, which is not equal to the data synchronization time point. The data synchronization time step $\Delta t_{s}$ is predefined and assumed to be fixed in the whole simulation, which means $\Delta t_{s}=t_{1}-t_{0}=\cdots=t_{n+1}-t_{n}$. $\Delta t_{s}$ should be a multiplier of the simulator time step size $\Delta t_{2}$ to avoid missing of the data synchronization point. Therefore, $\Delta t_{s}=N \Delta t_{2}$, and $N \in \mathbb{N}$, where $\mathbb{N}$ is natural numbers.

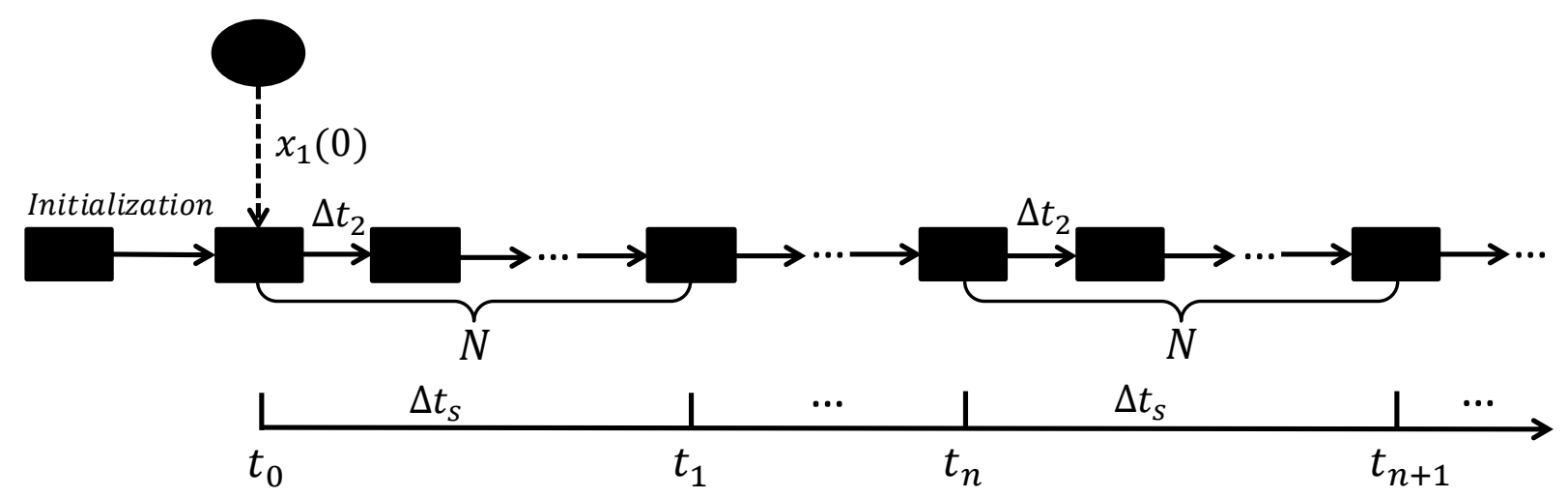

Figure 3 Schematic of one-way static coupling 
There are two typical applications of this scheme regarding the coupling between BES and CFD. First, BES can give the meteorological data to CFD as boundary condition and CFD calculates the airflow distribution in the buildings. In this case, BES is not required to be launched to perform any simulation. Second, CFD can determine the local weather data (pressure, velocity, temperature) in a microclimate or near the building (i.e., a community) and BES can then use the weather data to predict the ventilation performance of the natural ventilation in the building (Wang 2007). In this case, CFD, which is corresponding to simulator 1 in Figure 3, should perform a steady-state simulation. Surely, if the outdoor weather is dynamically changing, for example, when the simulation period is one year, this scheme which assumes that the local weather is not changing is not valid.

2) Two-way Static Scheme

Compared to the one-way static scheme, two-way static scheme requires a mutual data exchange at $t_{0}$ (Figure 4). Upon receiving the data $x_{2}(0)$ from simulator 2 , simulator 1 is called to perform a steady-state simulation and sends the data $x_{1}(0)$ back to simulator 2 . Then, assuming that $x_{1}$ is not changing over the whole simulation, simulator 2 performs a dynamics simulation. The procedure of the scheme is as follows:

Step 0: Initialize simulator 2 and set time step size as $\Delta t_{2}$. Send $x_{2}(0)$ to simulator 1 .

Step 1: Simulator 1 is called to generate a new state by performing a steady-state simulation with exchanged data $x_{2}(0)$ from simulator 2 :

$$
x_{1}(0)=f_{1}\left(x_{2}(0)\right)
$$

Step 2: Receiving data $x_{1}(0)$ from simulator 1 , simulator 2 generates an internal state after $j$ time step calculation as:

$$
x_{2}\left(t_{0}+j \Delta t_{2}\right)=f_{2}\left(x_{2}\left(t_{0}+(j-1) \Delta t_{2}\right), x_{1}(0)\right)
$$

Step 3: If $j=$ Last Step stop, else go to Step 2. 


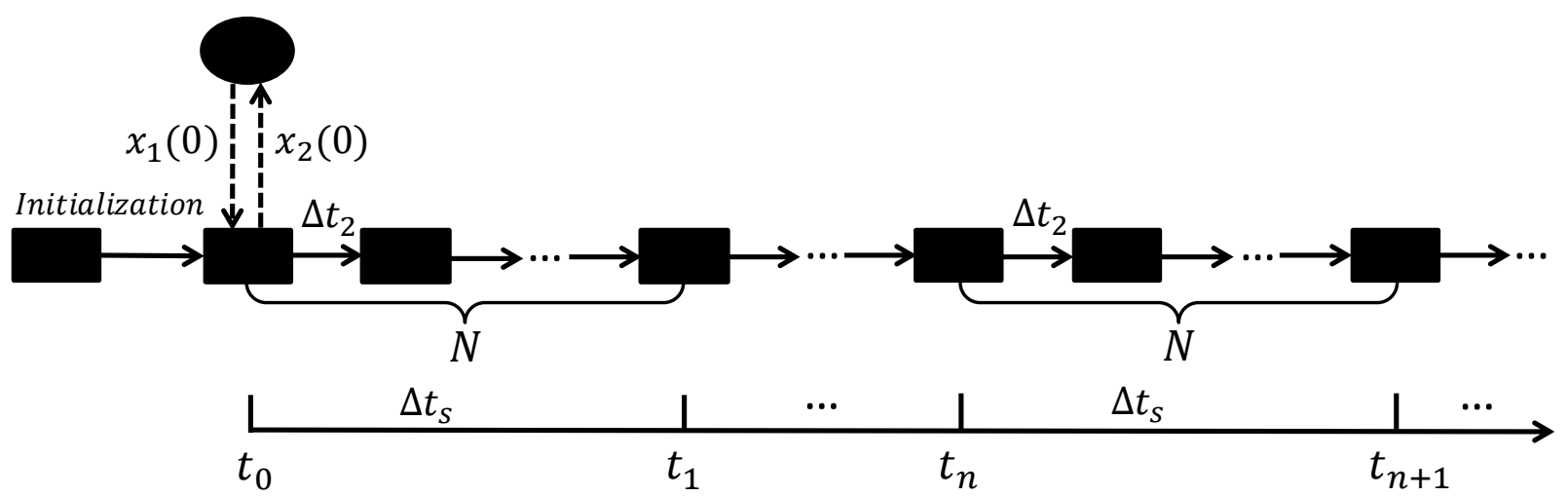

Figure 4 Schematic of two-way static coupling

This scheme is generally used in the situations where the domain simulated by either of the simulator does not dramatically change during the whole simulation time. For example, Zhai et al. (2002) applied this scheme to simulate an indoor auto-racing complex in Pittsburgh for 3 hours in a typical summer design day. BES first calculates the surface temperature and cooling load and sends them to CFD as boundary conditions. CFD then calculates the temperature distribution and sends the CHTC and temperature at fluid cells adjacent to the envelopes back to BES for 3 hours' simulation. In this case, given that a steady-state CFD simulation costs about 10 hours to be completed, a static coupling scheme seems a feasible option compared to a dynamic one.

\subsubsection{Dynamic Data Synchronization Schemes}

1) One-way Dynamic Scheme

Compared to the one-way static scheme, one-way dynamic scheme has multiple data exchange, which happens at an interval of $\Delta t_{s}$. As shown in Figure 5 , simulator 1 sends data $x_{1}$ to simulator 2 , while both simulator 1 and simulator 2 perform transient simulations. In this scheme and the rest schemes, we assume $\Delta t_{s}=M \Delta t_{1}=N \Delta t_{2}$, and $M, N \in \mathbb{N}$, where $\mathbb{N}$ is natural numbers. The procedure of the scheme is as follows:

Step 0: Initialize simulator 1 and simulator 2 and set time step size as $\Delta t_{1}, \Delta t_{2}$, respectively.

Step 1: If the synchronization point $t_{n+1}$ is not reached, compute new internal states: 


$$
\begin{aligned}
& x_{1}\left(t_{n}+i \Delta t_{1}\right)=f_{1}\left(x_{1}\left(t_{n}+(i-1) \Delta t_{1}\right)\right) \\
& x_{2}\left(t_{n}+j \Delta t_{2}\right)=f_{2}\left(x_{2}\left(t_{0}+(j-1) \Delta t_{2}\right), x_{1}(n)\right)
\end{aligned}
$$

Where $i, j \in \mathbb{N}$, and $i<M, j<N$

Step 2: If the synchronization point $t_{n+1}$ is reached, compute the exchanged data $x_{1}(n+1)$ :

$$
x_{1}(n+1)=x_{1}\left(t_{n}+M \Delta t_{1}\right)=f_{1}\left(x_{1}\left(t_{n}+(M-1) \Delta t_{1}\right)\right)
$$

Step 3: If $t_{n+1}=t_{\text {end }}$ stop, else go to Step 1 .

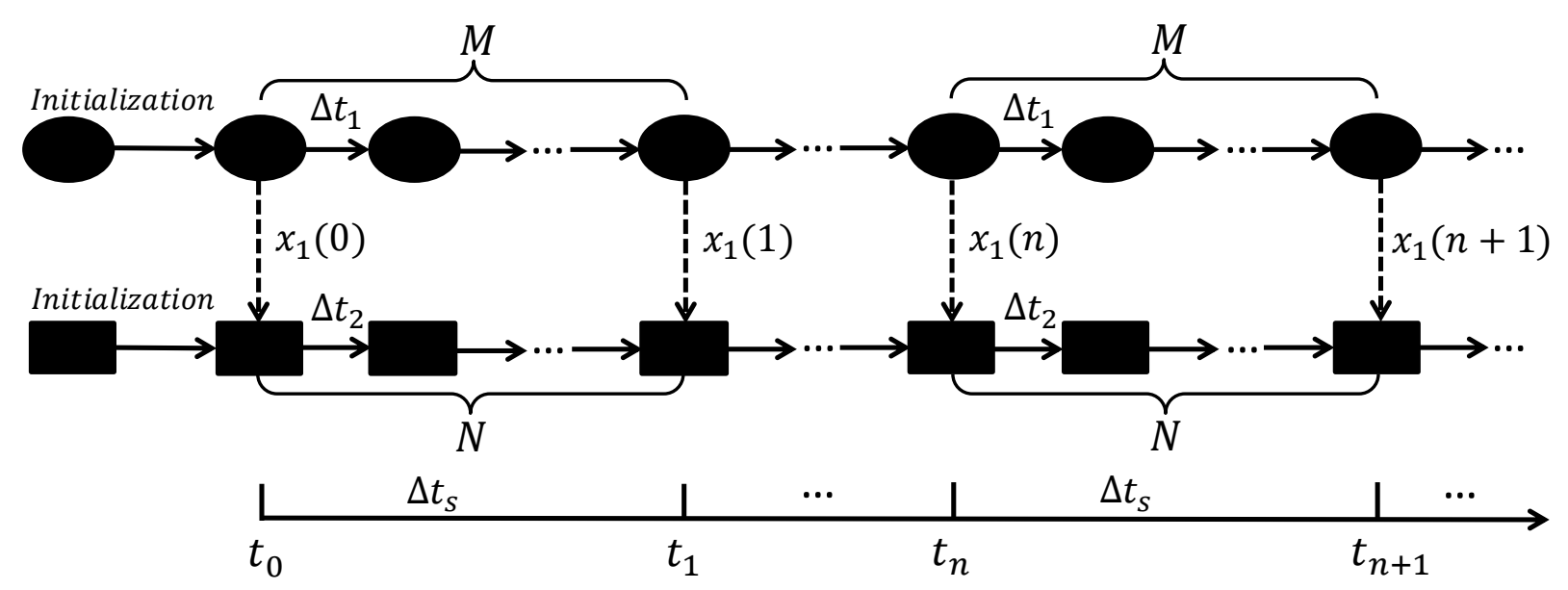

Figure 5 Schematic of one-way dynamic coupling

Novoselac (2004) compared different exchanged data for one-way dynamic coupling scheme. BES can give either heat flux at the envelope interior surfaces or heat flux through the wall to CFD. If CFD with a logwall wall function is used, they recommended that BES gives heat flux to CFD to avoid inaccurate heat flux calculation. Generally, compared to two-way coupling, one-way coupling can reduce the time cost for data exchange and may impose little risk to the stability of the coupled simulation. Given the fact that the status of HVAC system (simulated by BES) and indoor airflow (simulated by CFD) are fully coupled, one-way coupling can be used if one domain is not sensitive to the changes in the other domain. Otherwise, a twoway coupling scheme should be considered.

2) Quasi-Dynamic Scheme 
Quasi-dynamic synchronization is a two-way dynamic coupling scheme, in which BES and CFD mutually exchange data one time in a data synchronization time step. Quasi-dynamic scheme can be further divided into loose quasi-dynamic scheme and cross quasi-dynamic scheme (Trčka et al. 2009). In the loose quasidynamic scheme, BES and CFD run sequentially, which means one program would run only when the exchanged data from the other is available. In the cross quasi-dynamic scheme, BES and CFD can run in parallel and perform data exchange simultaneously at the data synchronization time point.

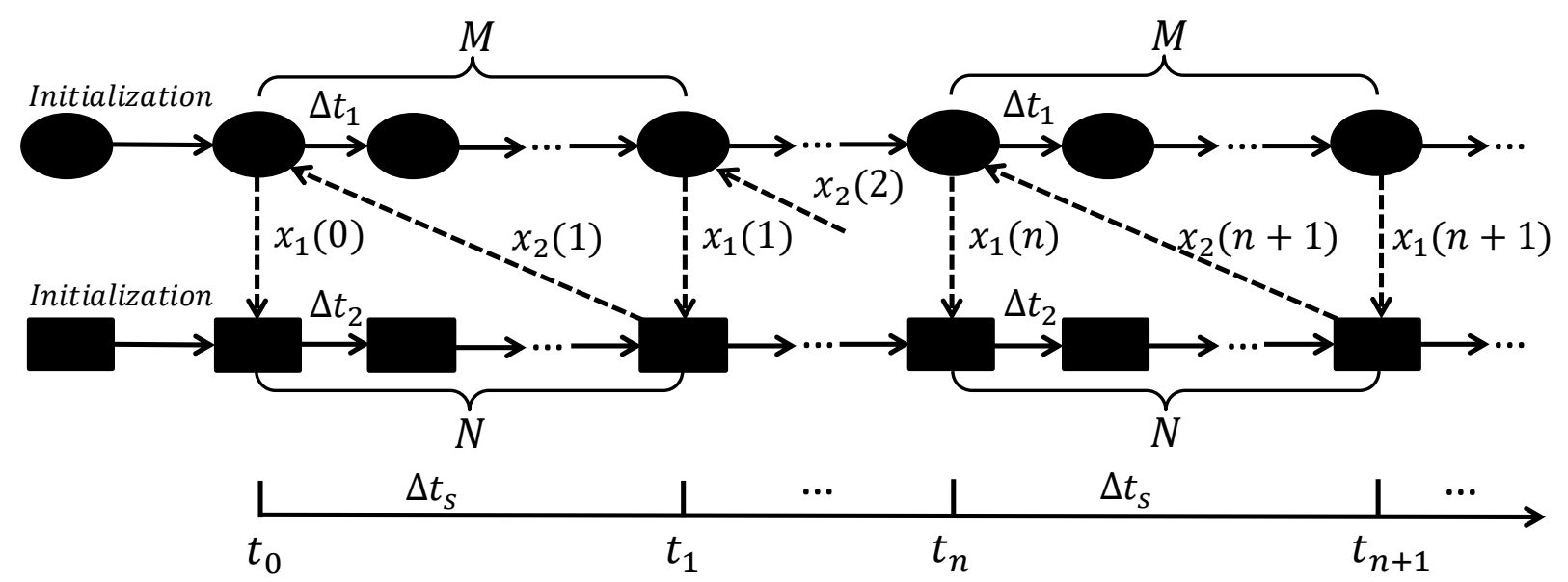

Figure 6 Schematic of loose quasi-dynamic coupling

The workflow of loose quasi-dynamic scheme is shown in Figure 6 . After initialization, at $t_{0}$, simulator 1 gives exchanged data $x_{1}(0)$ to simulator 2 and then holds until exchanged data from simulator 2 is available. Upon receiving $x_{1}(0)$ simulator 2 starts and marches on in time and sends out the exchanged data $x_{2}(1)$ to simulator 1 at the data synchronization time point $t_{1}$.While simulator 1 resumes to run from $t_{0}$ to $t_{1}$ after receiving $x_{2}(1)$, simulator 2 will not run until simulator finishes and sends out exchanged data $x_{1}(1)$. This process is repeated till the end of the simulation. Note that simulator 1 can perform steady state simulation. The procedure of the scheme is as follows:

Step 0: Initialize simulator 1 and simulator 2 and set time step size as $\Delta t_{1}, \Delta t_{2}$, respectively. 
Step 1: Simulator 2 start running while simulator 1 halts and wait for input from simulator 1.

Step 2: Receiving the input data $x_{1}(n)$ from simulator 1 , if the synchronization point $t_{n+1}$ is not reached in simulator 2, compute new internal state:

$$
\begin{aligned}
& x_{2}\left(t_{n}+j \Delta t_{2}\right)=f_{2}\left(x_{2}\left(t_{0}+(j-1) \Delta t_{2}\right), x_{1}(n)\right) \\
& \text { Where } j \in \mathbb{N} \text {, and } j<N
\end{aligned}
$$

Step 3: If the synchronization point $t_{n+1}$ is reached in simulator 2 , compute the exchanged data $x_{2}(n+1):$

$$
x_{2}(n+1)=x_{2}\left(t_{n}+N \Delta t_{2}\right)=f_{2}\left(x_{2}\left(t_{0}+(N-1) \Delta t_{2}\right), x_{1}(n)\right)
$$

Step 4: Receiving the input data $x_{2}(n+1)$ from simulator 2 , if the synchronization point $t_{n+1}$ is not reached in simulator 1 , compute new internal state:

$$
\begin{aligned}
& x_{1}\left(t_{n}+i \Delta t_{1}\right)=f_{1}\left(x_{1}\left(t_{n}+(i-1) \Delta t_{1}\right), x_{2}(n+1)\right) \\
& \text { Where } i \in \mathbb{N} \text {, and } i<M
\end{aligned}
$$

Step 5: If the synchronization point $t_{n+1}$ is reached in simulator 1 , compute the exchanged data $x_{1}(n+1):$

$$
x_{1}(n+1)=x_{1}\left(t_{n}+M \Delta t_{1}\right)=f_{1}\left(x_{1}\left(t_{n}+(M-1) \Delta t_{1}\right), x_{2}(n+1)\right)
$$

Step 6: If $t_{n+1}=t_{\text {end }}$ stop, else go to Step 2 .

The workflow of cross quasi-dynamic scheme is shown in Figure 7. Two simulators exchange data at data synchronization time point and then run simultaneously to the next point. For example, after initialization, at $t_{0}$, simulator 1 sends exchanged data $x_{1}(0)$ to simulator 2 while simulator 2 sends exchanged data $x_{2}(0)$ to simulator 1 . Once data exchange finishes, they resume to run at the same time to the next data synchronization point $t_{1}$. Note that two simulators should run transient simulations. The procedure of this scheme is as follows:

Step 0: Initialize simulator 1 and simulator 2 and set time step size as $\Delta t_{1}, \Delta t_{2}$, respectively. 
Step 1: Simulator 1 and simulator 2 are running in parallel.

Step 2: If the synchronization point $t_{n+1}$ is not reached, compute new internal states:

$$
\begin{aligned}
& x_{1}\left(t_{n}+i \Delta t_{1}\right)=f_{1}\left(x_{1}\left(t_{n}+(i-1) \Delta t_{1}\right), x_{2}(n)\right) \\
& x_{2}\left(t_{n}+j \Delta t_{2}\right)=f_{2}\left(x_{2}\left(t_{0}+(j-1) \Delta t_{2}\right), x_{1}(n)\right) \\
& \text { Where } i, j \in \mathbb{N}, \text { and } i<M, j<N
\end{aligned}
$$

Step 3: If the synchronization point $t_{n+1}$ is reached, compute the exchanged data $x_{1}(n+1)$ and $x_{2}(n+1):$

$$
\begin{aligned}
& x_{1}(n+1)=x_{1}\left(t_{n}+M \Delta t_{1}\right)=f_{1}\left(x_{1}\left(t_{n}+(M-1) \Delta t_{1}\right), x_{2}(n)\right) \\
& x_{2}(n+1)=x_{2}\left(t_{n}+N \Delta t_{2}\right)=f_{2}\left(x_{2}\left(t_{0}+(N-1) \Delta t_{2}\right), x_{1}(n)\right)
\end{aligned}
$$

Step 4: If $t_{n+1}=t_{\text {end }}$ stop, else go to Step 2.

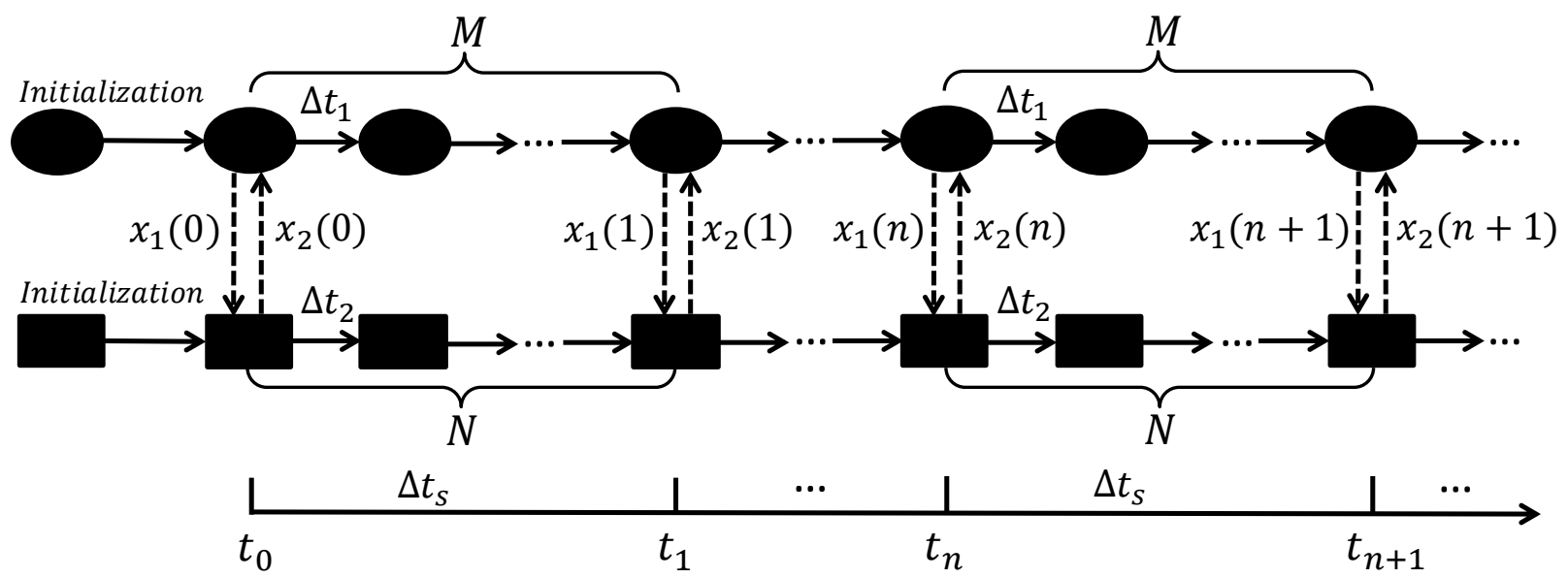

Figure 7 Schematic of cross quasi-dynamic coupling

Zhai et al. (2002) used the loose quasi-dynamic scheme to coupled BES and CFD to study heating load in an office with VAV. BES will resume to run until CFD sends back the CHTC and adjacent-to-wall fluid 
temperature. Novoselac (2004) used the similar approach to study the cooling load of an office. Similarly, Du et al. (2015) coupled BES and CFD in a loose quasi-dynamic scheme and applied optimization to study the thermostat placement. CFD simulates the thermal environment and extracts the temperature at thermostat to BES, which then simulates the control of VAV system. Since CFD simulation is usually timeconsuming, loose quasi-dynamic is relatively more popular as CFD can be called few times to run a steady state simulation to save computation time. With a significantly faster solver for CFD, Zuo, Wetter, et al. (2016) coupled BES and CFD in the cross dynamic scheme and studied the control of HVAC system. BES and CFD exchange information at a predefined interval and then run at the same time.

Quasi-dynamic coupling scheme can achieve a balance between accuracy and computation speed. Thus, the determination of data synchronization time step size is critical. On one hand, with a shorter data synchronization time step, the result accuracy can generally be improved with a penalty of increased computation time. On the other hand, with larger data synchronization time step size, the computation speed can be improved with a sacrifice in result accuracy. Especially when a loose coupling scheme in which steady state CFD simulation is performed, the number of CFD call can be proportionally decreased with the increase of data synchronization time step. Note that Our literature review did not identify a strict rule to set $\Delta t_{s}$, and users have a certain level of freedom to do that as long as $\Delta t_{s}$ is comparable to the time scale of the simulated physical phenomena. For example, if heat flux is to be determined by co-simulation (Zhai et al. 2002), $\Delta t_{s}$ can be set as large as 1 hours. If control of cooling system is to be studied using cosimulation (Zuo, Wetter, et al. 2016), $\Delta t_{s}$ can be set as large as small as 4 seconds. Novoselac (2004) compared the result accuracy of predicted cooling load for an office by varying the data synchronization time step and found that with a one-hour time step size the prediction error can reach $30 \%$ while a 10 minute time step size can produce comparable results as benchmark, when outside condition is dynamically changing.

3) Fully Dynamic Scheme

Fully dynamic scheme requires iterations at each data synchronization step until both simulations are converged. Thus, it is theoretically the most accurate scheme and may generate the same results as the 
internal coupling, when the data synchronization time step size is infinitely small. It can have a simplified form (Figure 8), in which one program runs transient simulation and the other runs steady state simulation, and a rigorous form (Figure 9), in which both programs run transient simulations. The process is very much like quasi-dynamic coupling scheme except that several rounds of iterations are needed (box in dotted line in the figures).

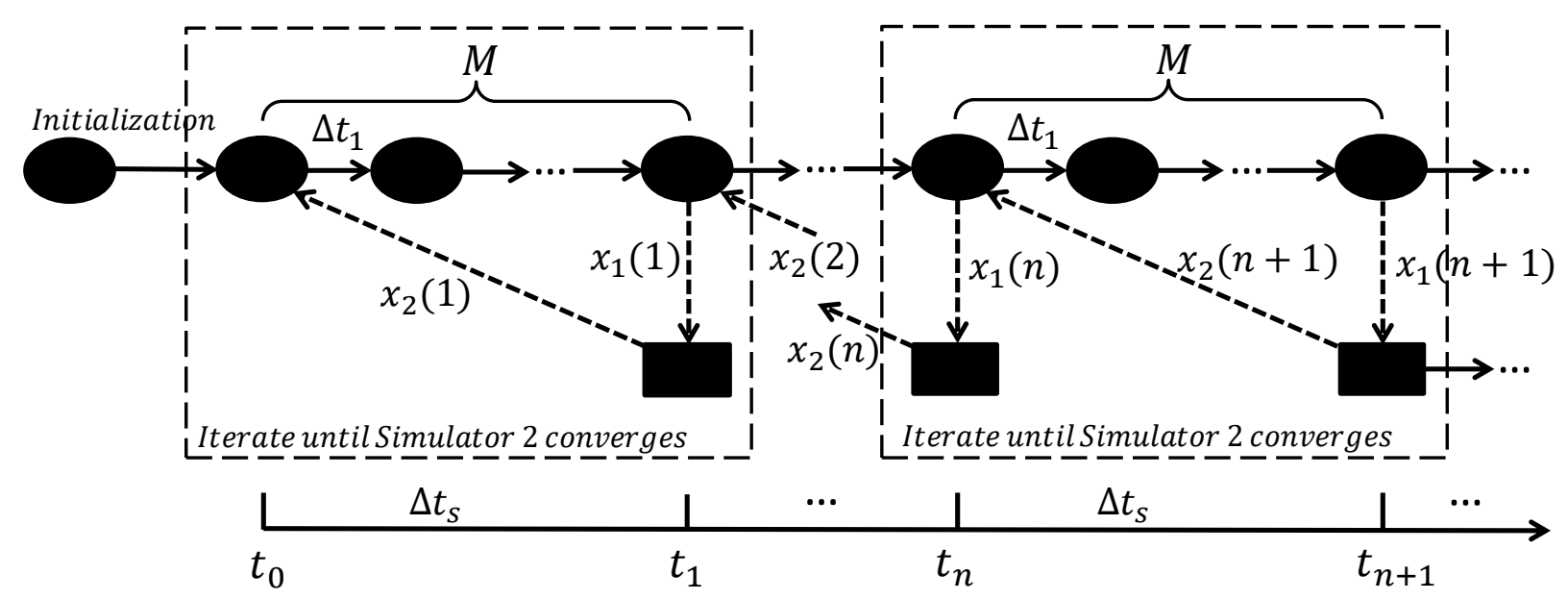

Figure 8 Schematic of fully dynamic coupling in a simplified form

The detailed procedures of a simplified form of fully dynamic scheme are explained as follows:

Step 0: Initialize simulator 1 and set time step size as $\Delta t_{1}$.

Step 1.1: Simulator 1 starts running, if the synchronization point $t_{n+1}$ is not reached, compute new internal state:

$$
\begin{aligned}
& x_{1}\left(t_{n}+i \Delta t_{1}\right)=f_{1}\left(x_{1}\left(t_{n}+(i-1) \Delta t_{1}\right)\right) \\
& \text { Where } i \in \mathbb{N} \text {, and } i<M
\end{aligned}
$$

Step 1.2: If the synchronization point $t_{n+1}$ is reached for the first time, compute the exchanged data $x_{1}^{0}(n+1)$, and sent it to simulator 2 to compute exchanged data $x_{2}^{0}(n+1)$ :

$$
x_{1}^{0}(n+1)=x_{1}\left(t_{n}+M \Delta t_{1}\right)=f_{1}\left(x_{1}\left(t_{n}+(M-1) \Delta t_{1}\right), x_{2}(n)\right)
$$




$$
x_{2}^{0}(n+1)=f_{2}\left(x_{1}^{0}(n+1)\right)
$$

Note that super script in $x_{1}^{0}$ and $x_{2}^{0}$ represents number of iterations.

Step 2: Reset the time in simulator 1 to $t_{n}$ and start the simulator 1 . Suppose it is $k$ iteration.

Step 3.1: If the synchronization point $t_{n+1}$ is not reached in simulator 1 , compute the internal state:

$$
x_{1}^{k}\left(t_{n}+M \Delta t_{1}\right)=f_{1}\left(x_{1}^{k}\left(t_{n}+(M-1) \Delta t_{1}\right), x_{2}^{k-1}(n+1)\right)
$$

Step 3.2: If the synchronization point $t_{n+1}$ is reached in simulator 1 , compute the internal state $x_{1}^{k}(n+1)$ and sent it to simulator 2 to compute exchange data $x_{2}^{k}(n+1)$ :

$$
\begin{aligned}
& x_{1}^{k}(n+1)=x_{1}^{k}\left(t_{n}+M \Delta t_{1}\right)=f_{1}\left(x_{1}^{k}\left(t_{n}+(M-1) \Delta t_{1}\right), x_{2}^{k-1}(n+1)\right) \\
& x_{2}^{k}(n+1)=f_{2}\left(x_{1}^{k}(n+1)\right)
\end{aligned}
$$

Step 4: If $x_{2}^{k}(n+1) \approx x_{2}^{k-1}(n+1)$ go to Step 5, else repeating Step 2.

Step 5: If $t_{n+1}=t_{\text {end }}$ stop, else go to Step 1.1.

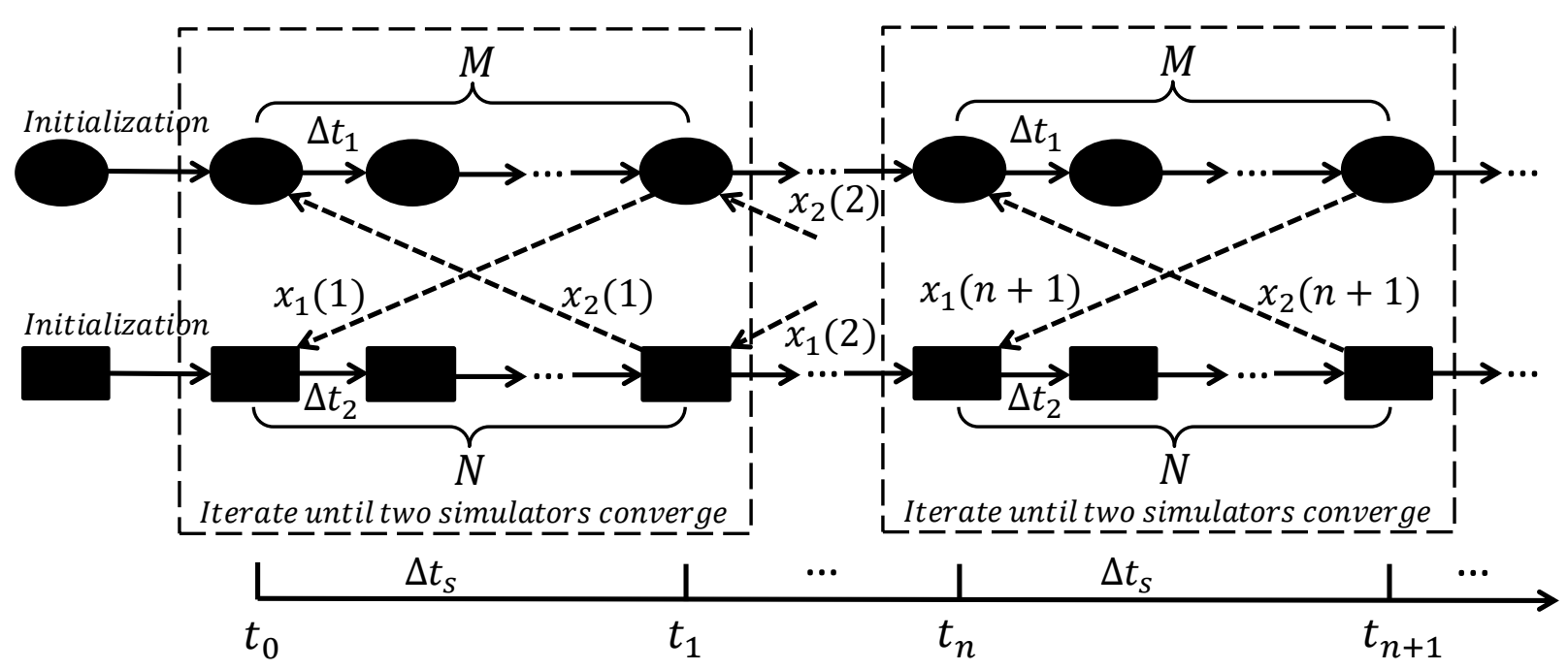

Figure 9 Schematic of fully dynamic coupling in a rigorous form 
The detailed procedures of a rigorous form of fully dynamic scheme are explained as follows:

Step 0: Initialize simulator 1 and simulator 2 and set time step size as $\Delta t_{1}, \Delta t_{2}$, respectively.

Step 1.1: Simulator 1 and simulator 2 start running in parallel. if the synchronization point $t_{n+1}$ is not reached, compute new internal states:

$$
\begin{aligned}
& x_{1}\left(t_{n}+i \Delta t_{1}\right)=f_{1}\left(x_{1}\left(t_{n}+(i-1) \Delta t_{1}\right)\right) \\
& x_{2}\left(t_{n}+j \Delta t_{2}\right)=f_{2}\left(x_{2}\left(t_{n}+(j-1) \Delta t_{2}\right)\right) \\
& \text { Where } i, j \in \mathbb{N} \text {, and } i<M, j<N
\end{aligned}
$$

Step 1.2: If the synchronization point $t_{n+1}$ is reached for the first time, compute the exchanged data $x_{1}^{0}(n+1)$, and sent it to simulator 2 to compute exchange data $x_{2}^{0}(n+1)$ :

$$
\begin{aligned}
& x_{1}^{0}(n+1)=x_{1}\left(t_{n}+M \Delta t_{1}\right)=f_{1}\left(x_{1}\left(t_{n}+(M-1) \Delta t_{1}\right)\right) \\
& x_{2}^{0}(n+1)=x_{2}\left(t_{n}+N \Delta t_{2}\right)=f_{2}\left(x_{1}\left(t_{n}+(N-1) \Delta t_{2}\right)\right)
\end{aligned}
$$

Note that super script in $x_{1}^{0}$ and $x_{2}^{0}$ represents number of iterations.

Step 2: Reset the time in two simulators to $t_{n}$ and start the simulators. Suppose it is $k$ iteration.

Step 3.1: If the synchronization point $t_{n+1}$ is not reached, compute the internal states:

$$
\begin{aligned}
& x_{1}^{k}\left(t_{n}+M \Delta t_{1}\right)=f_{1}\left(x_{1}^{k}\left(t_{n}+(M-1) \Delta t_{1}\right), x_{2}^{k-1}(n+1)\right) \\
& x_{2}^{k}\left(t_{n}+M \Delta t_{1}\right)=f_{1}\left(x_{2}^{k}\left(t_{n}+(N-1) \Delta t_{2}\right), x_{1}^{k-1}(n+1)\right)
\end{aligned}
$$

Step 3.2: If the synchronization point $t_{n+1}$ is reached, compute the internal state $x_{1}^{k}(n+1)$ and $x_{2}^{k}(n+1):$

$$
\begin{aligned}
& x_{1}^{k}(n+1)=x_{1}^{k}\left(t_{n}+M \Delta t_{1}\right)=f_{1}\left(x_{1}^{k}\left(t_{n}+(M-1) \Delta t_{1}\right), x_{2}^{k-1}(n+1)\right) \\
& x_{2}^{k}(n+1)=x_{2}^{k}\left(t_{n}+N \Delta t_{2}\right)=f_{2}\left(x_{2}^{k}\left(t_{n}+(M-1) \Delta t_{1}\right), x_{2}^{k-1}(n+1)\right)
\end{aligned}
$$

Step 4: If $x_{2}^{k}(n+1) \approx x_{2}^{k-1}(n+1)$ go to Step 5, else repeating Step 2. 
Step 5: If $t_{n+1}=t_{\text {end }}$ stop, else go to Step 1.1.

Compared to the quasi-dynamic scheme, the fully dynamic scheme may perform better in terms of accuracy. Requiring iterations to achieve converged results for each data synchronization time step, the results of fully dynamic scheme are much less sensitive to the time step size. Users must set a reasonable error criteria to terminate iterations, to balance the computational cost and accuracy. One major challenge of applying fully dynamic scheme is that the selection of exchanged data may impact the convergence, stability and computing speed of the coupled simulation. For example, Zhai and Chen (2003) compared three sets of exchanged data between BES and CFD to calculate the thermal load of the building, and found that sending the enclosure interior surface temperature from BES to the CFD and returning the CHTC and indoor air temperature gradients to from CFD to BES can achieve unconditional stability and a faster computation speed. Similarly, Wang (2007) compared thee sets of exchanged data between BES and CFD to simulate the airflow distribution in a building, and concluded that exchanging total pressure between BES and CFD is unconditional stable.

\subsection{Coupled Simulation Implementations}

This section introduces the software realization of the data synchronization schemes discussed in Section 2.3. Three approaches to implement the coupling between BES and CFD are explained: customized interfaces, middleware, and standard interfaces.

\subsubsection{Customized Interfaces}

Customized interfaces are a set of import and export interfaces defined by users to facilitate the data exchange between two programs. Based on the problem that the coupled simulation is targeted to resolve, users can define the exchanged data and then design the interfaces accordingly. Figure 10 illustrates the coupling between BES and CFD using the customized interfaces. For ease of explaining, we distinguish the interface to import and export exchanged data. In real applications, it may be combined in one single interface. When the data synchronization time point is reached in two simulators, the interface reads the exchange data from the engine and write the data to the other simulator. Meanwhile, the interface also 
reads the exchanged data sent from the other simulator and updates the states in the simulation engine.

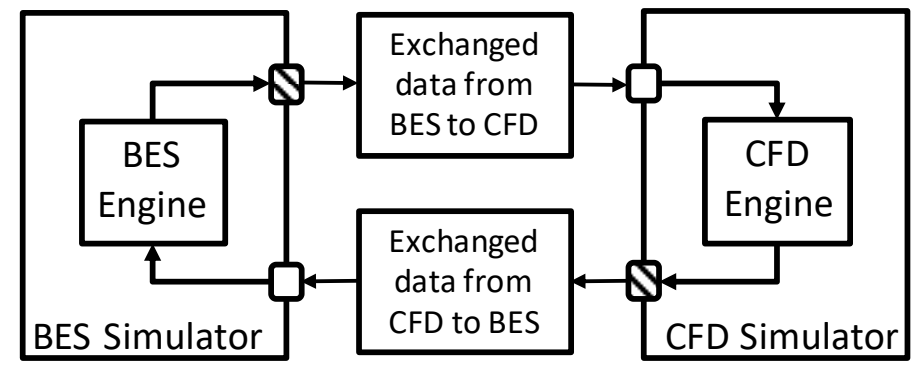

Quser-defined Export Interface $\square$ User-defined Import Interface

Figure 10 Schematic of coupled simulation implementation using customized interface

The exchanged data can be either stored in a text-based file (Fan and Ito 2012) or shared memory in the random-access memory (Zuo et al. 2014). The exchanged data can be saved into a text file as long as the access to the state of simulation engine is valid (either the user can inquiry the engine for the state or the engine can output its state if being requested). This method is relatively easy and stable as the users are not required to handle and modify the engine. However, the drawback is the overhead of data communication in writing and reading the text files. Especially when the data synchronization time step size is small and the size of exchanged data is large, the overhead can be a bottleneck for speeding the coupled simulation. To resolve this limitation, another way is to store the exchanged data in a memory buffer shared by BES and CFD. Using this method, the speed of writing and reading data can be significantly faster and thus help reduce the overhead. In order to share the memory between BES and CFD, the users are expected to have the source codes of both simulators and be knowledgeable to carry out a successful implementation. Thus, the method based on shared memory requires more efforts and expertise in the implementation, and it poses additional risk in robustness of the simulation that is derived from the run-time management of the shared memory.

Without a dedicated program to coordinate two simulators, the coupled simulation is usually carried out 
in a master-slave mode. In this mode, one of the simulator (act as master) controls the whole process of the coupled simulation: define data synchronization time point and check if that time point is reached, call the other simulator (act as slave), control the order of data exchange, and terminate the simulation. The control information from master can be transferred to the slave in the exchanged data, including: next time point to synchronize data, flag to stop or resume slave simulation, flag to show if new exchanged data is ready, and flag to stop the slave. Using the master-slave mode, it is theoretically feasible for the users to implement all the data synchronization schemes introduced in Section 2.3. However, it can be challenging to perform the fully dynamic coupling, as extensive programming and modifying of the simulation engine is required to achieve resetting of the time.

The approach of customized interfaces is straightforward and provides a certain level of controllability to the users, nevertheless, this approach may be time-consuming to implement if compared to the other approaches introduced in the rest of the section. Moreover, the maintainability and expandability of this approach is limited, as the users may need to reprogram the interface regarding a new application or using a new simulator. Lastly, this approach can hardly support running the simulators on different cores of the computer to take advantage of the computation power of the CPU and distributed computing on clusters of computers.

\subsubsection{Middleware}

Middleware connects two or more simulators through its internal actors which can exchange data mutually. The actor is defined as an intermediate agent to connect external simulators, such as BES and CFD program. The middleware can control the data synchronization between two simulators by directly controlling the data exchange between actors. As shown in Figure 11, BES and CFD are coupled through the middleware by being connected to actor 1 and actor 2, respectively. Take the one-way dynamic coupling (BES runs continuously and CFD runs intermittently) as example, at the data synchronization time point, the middleware will send a signal to BES through actor 1 by reading the exchanged data and hold the BES simulation while waiting for the data from CFD. Meanwhile, the middleware will fire off the CFD simulator with the exchanged data passed from actor 1 to actor 2. Once CFD simulation finishes, the exchanged data 
will be read back to actor 2 and eventually passed to BES through actor 1. Receiving the exchanged data, BES simulation resumes and keeps on till the next data synchronization point.

Compared to the master-slave mode using in customized interfaces, the middleware serves as the coordinator and controller to the coupled simulation. It can fire off or hold either simulator based on the intended data synchronization scheme. The middleware can also support the mathematical operation on the exchanged data, such as integration over the time. Last, the middleware can provide timely run-time visualization of the exchange information.

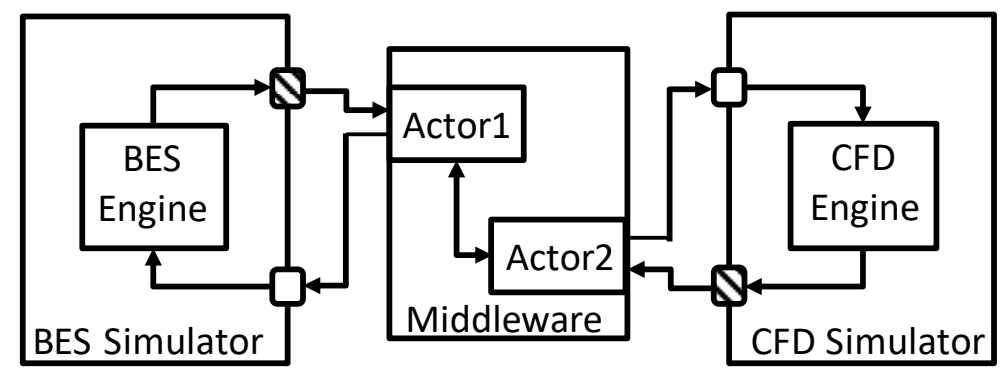

Q Middleware Compatible Export Interface

$\square$ Middleware Compatible Import Interface

Figure 11 Schematic of middleware interface

Based on how actors are internally synchronized, there are mainly two categories of the middleware implementation. One is to enable data exchange between actors within a software program. Two examples of this category are Building Controls Virtual Test Bed (BCVTB) (Wetter 2011) and Agent and Artefact for Multiple Models (AA4MM) (Siebert et al. 2010). BCVTB was developed based on Ptolemy II and used extensively for coupled simulation in buildings (Trčka et al. 2009; Pang et al. 2011). BCVTB provides interfaces to connect to some of the popular BES programs, such as EnergyPlus, TRNSYS, ESP-r, etc. The actors are connected to the simulators through Berkeley Software Distribution socket based on TCP/IP. This feature enables BCVTB to connect simulators running on different computers to take advantage of the distributed computing techniques. The actors are synchronized using a Model of Computation, one of which is synchronous data flow (SDF). Using SDF director, the actor will calculate a predefined number of output 
tokens when the input token on each input port is available. AA4MM is a similar architecture but targeted for a broader coupled simulation application. In the context of AA4MM, the actor is equivalent to model agent. In AA4MM, the coupling-artefact module is used to synchronize data between actors (model agents). A software built on AA4M paradigm named MECSYCO is available (Camus et al. 2016). Compared to AA4M, BCVTB is more popular in coupled simulation regarding buildings. However, there are few limitations of using BCVTB to coupling BES and CFD:

1. As of October 2017, BCVTB does not provide an interface to commercial CFD programs. It is necessary for users to develop a code to bridge the CFD program and BCVTB, such as FLOW+ which is used to connect FLUENT to BCVTB (Zhang et al. 2012b).

2. Developing interfaces to BCVTB can be challenging for normal users. This is mainly because it is required to understand the BSD before the implementation of the BSD client, as the simulator (BES, CFD) is connected to the actor through the BSD.

3. Only quasi-dynamic coupling scheme is supported. The fully dynamic coupling scheme that requires iteration between simulators is not supported.

4. Only fixed data synchronization step size is supported.

The other one is to enable data exchange between actors through Information Exchange Bus (IEB). The example of this category is VOLTTRON, which is an open source and agent-based platform developed by Pacific Northwest National Laboratory to support transactional energy activities (Akyol et al. 2015). VOLTTRON was first proposed to study the control of buildings, power systems and smart grid that involve multiple components proactively interacted with each other, not mainly for the coupled simulation purpose in buildings (Akyol et al. 2012; Haack et al. 2013; Haack et al. 2016). However, some characteristics of VOLTTRON make it compelling to be used to couple BES and CFD. Note that in the context of VOLTTRON, actor is equivalent to agent.

1. IEB based on ZeroMQ provides fast communication between agents (Akyol et al. 2015). ZeroMQ supports sock-based application programming interface (API), which indicates that distributed computing on different computers can be used to expedite the coupled simulation between BES and CFD. ZeroMQ 
supports data exchange regardless of the programming language of BES and CFD which dramatically simplifies the interface development.

2. Existing agents or a user-defined agent can potentially act as masters to the coupled simulation between BES and CFD.

3. VOLTTRON does not require a fixed data synchronization time step size.

4. Like BCVTB, VOLTTRON supports hardware-in-the-loop. This can be used to validate the coupled simulation model.

\subsubsection{Standard Interfaces}

Standard interfaces are tool-independent interfaces to support direct connection between two simulators. As shown in Figure 12, BES and CFD are coupled directly through the standard interfaces without intermediate steps. Compared to previous approaches, this approach using standard interfaces is straightforward and easy-to-deploy if the interfaces are implemented by the software providers.

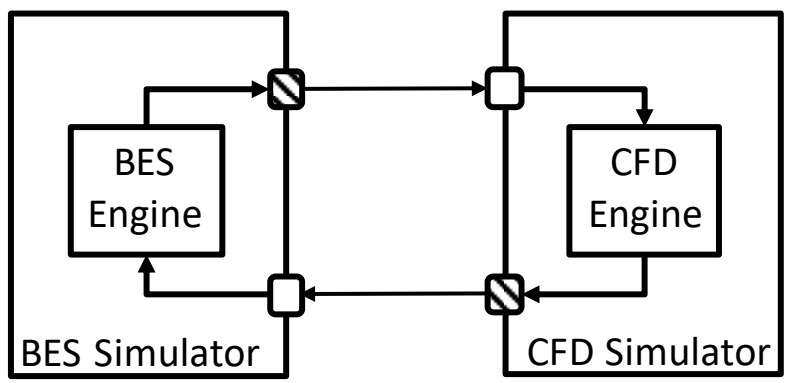

Q Standard Interface to Export

$\square$ Standard Interface to Import

Figure 12 Schematic of standard interface

One of the standard interfaces that are using in co-simulation in buildings is the Functional Mock-up Interface (FMI) (Blochwitz, Otter, Arnold, Bausch, Clauß, et al. 2011; Wetter and Van Treeck 2017). FMI is 
a tool-independent standard to support model exchange and co-simulation for dynamic simulation involving multiple physical domains. The FMI version 2.0 is currently available. Many BES programs have been developed to be compatible to FMI, such as EnergyPlus (Nouidui et al. 2014), TRNSYS (Widl et al. 2015) and Modelica-based modelling (Wetter et al. 2014). However, as of October 2017, there is neither official release nor third-party development of FMI to the commercial or open-source CFD programs. Nevertheless, there is a report discussing the coupling of EnergyPlus and Modelica using FMI to CFX (Ansys 2009) using a customized script-based interface (Wetter and Van Treeck 2017).

Figure 13 shows the procedure of internal and external coupling through FMI. Suppose a FMI implementation has been performed for CFD. Through the FMI for model exchange, CFD can be exported as Functional Mock-up Units (FMU), which is a zip file containing pre-compiled binary file (c-functions), a $\mathrm{XML}$ file, and possibly some resource files. For model exchange, the binary file only describes the mathematical representation of the system without a solver attached. The XML file provides description of the exchanged data, parameters, values for variable initialization. Then the CFD FMU is imported to the BES through the FMI. Afterward, the equations from CFD and BES are solved simultaneously using BES's solver, in a way identical to internal coupling scheme. On the other hand, a FMU of CFD can be exported for co-simulation, and in this case, the binary file has the mathematical representation of the system as well as its solver. The XML contains extra information regarding the co-simulation controls. Once being imported into the BES, the co-simulation (external coupling scheme) can perform in a master-slave mode by exchanging predefined data mutually. Since FMU from CFD has its own solver, it would not use the solver in BES. 


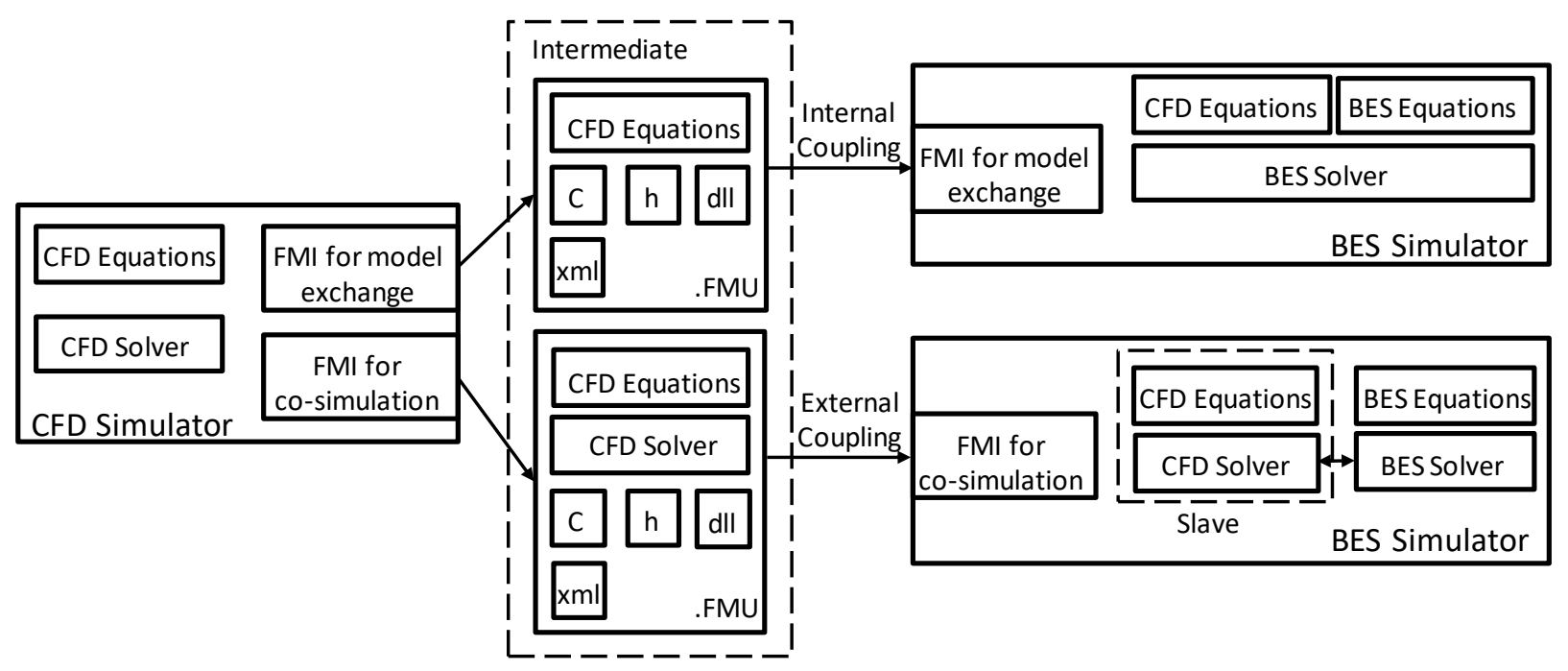

Figure 13 Schematic of coupled simulation implementation using FMI

BES can be used to serve as the master for the coupled simulation. However, the functionality is limited due to the difficulty to program the advanced master algorithm in BES. The middleware including BCVTB and $\mathrm{AA} 4 \mathrm{MM}$ can couple FMUs and control the coupled simulation process. However, they are not exclusively designed for FMUs and therefore not fully capable to take full advantage of FMUs, such as performing fully dynamic simulations. Researchers have developed dedicated master programs to facilitate co-simulation using FMUs, such as PyFMI (Andersson et al. 2016), MasterSIM (Institut für Bauklimatik 2017), and DACCOSIM (RISEGrid 2017), etc. Except for some normal handling of the FMUs, such as loading, initializing and coupling FMUs, the highlight of those masters is that they support fully dynamic coupling scheme by enable resetting the state for each FMU and error control for co-simulation that is critical for optimization study. For example, PyFMI which is compatible to FMI 2.0, has a class Master to control the co-simulation (Andersson et al. 2016). First, the fully dynamic coupling scheme is realizable in PyFMI because FMI 2.0 supports retrieving and restoring the internal state and separate initialization of FMU. As shown in Figure 14, after loading the FMU and during the simulation, PyFMI can have the access to the internal state of the FMU, and those states can be used to reinitialize the FMU during the iteration when fully dynamic coupling is applied. Second, it provides the option for users to specify either absolute or relative error tolerance if error control is turn on by using adaptive time step size in simulation. The error 
estimation in the simulation is based on Richardson extrapolation. The error estimation is associated to the difference by running one simulation using the data synchronization step size and two sequential simulations using half the time step size. Moreover, PyFMI has other features that are critical to applying co-simulation into real engineering application, such as parameter estimation, smoothing of the discontinuous inputs, etc.

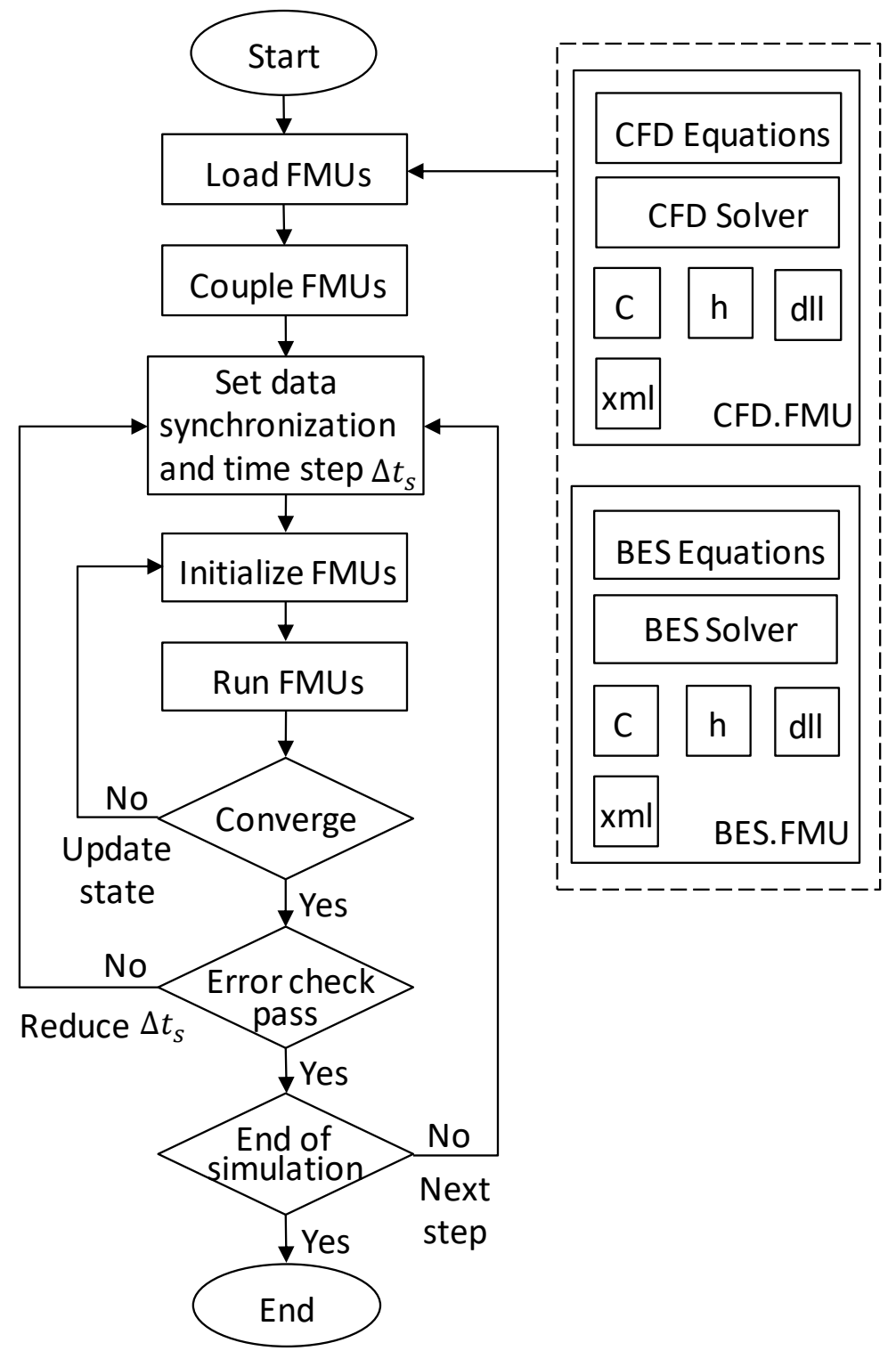

Figure 14 Workflow of co-simulation master for FMUs 


\section{Recent and Potential Co-Simulation Applications}

\subsection{Energy-Related Applications}

\subsubsection{Heating or cooling load calculation}

BES calculates cooling or heating load by simulating the heat and mass transfer process, such as the heat and mass balance method used in EnergyPlus. Many researches have demonstrated that coupling CFD can improve the accuracy of the building energy simulation (Clarke et al. 1995; Negrao 1995; Nielsen and Tryggvason 1998; Srebric et al. 2000; Beausoleil-Morrison 2000). For example, as mentioned in Section 2.1, CFD can provide BES the CHTC, which would impact the cooling or heating load calculation in BES.

The airflow model has great impact for BES on the heating and cooling load calculation. Han et al. (2015) coupled EnergyPlus and CONTAM to compare the building energy simulation with different air filtration estimation methods. They found the results of coupled simulation method are more accurate than that using default setting and database. Dols et al. (2015) employed coupled simulation to predict infiltration and exfiltration rates through the building entrance doors and their impact on the heating and cooling load. It was found that the use of vestibule could reduce the infiltration by $23 \%$ and the annually energy saving can be $1.4 \%$. Pan et al. (2010) coupled EnergyPlus and CFD to study the cooling load estimating method for atrium buildings. The CFD returns air temperature distribution to EnergyPlus based on boundary conditions received from EnergyPlus. It is found that the conventional well mixed airflow model will cause significant errors for cooling load and building energy calculation for atrium buildings with high glazing and a simplified model based on CFD results to describe stratified airflow can get fairly accurate estimated results for cooling load.

Weather data is also very important for BES to predict the cooling and heating load. Typical year weather data in the BES may not be accurate for a specific building in a community where a local microclimate that can be different from the climate in the weather station (Pisello et al. 2012). The CFD can 
predict the microclimate using the typical year weather data in BES, and with the updated weather data, the BES is expected to provide more accurate building energy simulation (Bouyer et al. 2011; Yi and Malkawi 2008). Allegrini and Carmeliet (2017) pointed out the energy demand for buildings and the thermal comfort and health for humans are strongly influenced by the local microclimate and employed an one-way coupled simulation between CFD and BES to estimate the effect of the new buildings on the local microclimate. Wang (2007) demonstrated that for a low-rise house, by feeding the weather data in a format of wind pressure profile from CFD to BES, the relative difference of airflow rates at openings between prediction and experiment measurements, can drop 2.14 times from the $30 \%$ by using standalone BES to $17 \%$.

\subsubsection{Design and performance evaluation of advanced indoor ventilation method}

Some ventilation schemes, such as personalized ventilation, displacement ventilation, and natural or hybrid ventilation are proposed to improve the indoor environment quality and reduce energy consumption. Unfortunately, BES with multizone airflow models are not well suitable for the simulation of non-uniformly distributed air in these cases.

With personalized ventilation, occupants can adjust the ventilation or air-conditioning according to his/her thermal sensation and preference for air quality. In the coupled simulation, CFD transfers to the BES the detailed information of local thermal comfort and temperature distribution around the occupants so that the ventilation rate can be adjusted to meet the requirement. Gao et al. (2006) coupled CFD and human thermal regulation model for a better performance assessment of personal ventilation in terms of thermal conform. Adding BES will also enable the performance assessment of personal ventilation in terms of energy consumption and controllability.

The displacement ventilation uses the temperature stratification to improve the thermal comfort while reduce energy consumption. It is difficult to estimate the indoor airflow distribution and actual building energy consumption by running BES alone since the stratified temperature distribution strongly impact the 
CHTC of exterior walls and voids empirical formula in BES (Zhai et al. 2002). CFD obtains temperature distribution on which correct $\mathrm{CHTC}$ can be calculated. Fed into updated $\mathrm{CHTC}$ and temperature distribution, BES calculates new interior surface temperature, the heat flux, inlet flow temperature. These parameters will be transferred to CFD as boundary conditions.

Natural ventilation, which is widely used in buildings for its cleanness and energy saving performance, is complicated and needs to be fully understood. Through coupled simulation, BES calculates the interior surface temperature and the heat flux in buildings. These parameters are transferred to CFD for obtaining the local air temperature distribution and CHTC which is further given to BES to run for next time step. Eventually the coupled simulation answers how many natural ventilation hours are usable and how much air-conditioning energy consumption can be avoid (Zhang et al. 2012b). The coupled simulation could simultaneously estimate building energy use, airflow and indoor air quality (IAQ) and evaluated the design for natural ventilation (Dols et al. 2016; Li and Li 2015; Liu et al. 2014).

\subsubsection{Thermal performance of double-skin facades, double-skin roofs and membrane}

A façade can bring away the solar energy by buoyance driven ventilation so that the room will not be heated (Pappas and Zhai 2008). BES can calculate the solar heat gain and cooling loads of the room. However, the thermal performance of the façade is highly impacted by the airflow inside and around the facade that is difficult to simulate by BES due to the limitation of its airflow model. By calculating the detailed flow phenomena for this complex geometry, CFD can provide a better thermal performance of the façade than that by the simple airflow model in BES (Zeng et al. 2012; Pappas and Zhai 2008; Fuliotto et al. 2010).

Villi et al. (2009) used the coupled simulation to study the performance of the wooden ventilated roof on thermal comfort and energy saving. Double-skin wooden ventilation roof takes advantage of Buoyancy ventilation in the cavity to bring away the heat gain through convective heat transfer. The height of the cavity is crucial for the roof design. CFD simulates the airflow and CHTC in the channel, as well as to develop 
correlation between the airflow and heat transfer coefficient. Then correlations will be used when BES is performed to simulate the annual air-conditioning load.

Membrane is favored since it can allow the most of daylighting passing through (Zhang et al. 2000). Devulder et al. (2007) studied the how membrane and its enclosed space reduce the air-conditioning load and improve the thermal comfort in the building using the coupled simulation. The airflow in the enclose space beneath the membrane is investigated by CFD. Its thermal performance, which is a key issue for this structure, will be obtained with the BES that simulates the solar radiations and the CFD that simulates the airflow.

\subsection{Control-Related Applications}

\subsubsection{Performance of the advanced air-conditioning methods}

The coupled simulation of CFD and BES can be used for evaluating new air-conditioning methods, such as underfloor cooling/heating, air-conditioning with energy recovery ventilation, stratified airconditioning for large space or atrium, and zonal relative humidity or temperature control. Underfloor heating with a top return can maintain comfortable thermal environment by using far more less energy than a ceiling based system (Wan and Chao 2002). Energy recovery ventilation is employed to further save the airconditioning energy. Obtaining the correct outlet air temperature is critical for predicting the heat exchange rate of energy recovery in the ventilation (Fan and Ito 2012). The underfloor heating lead to vertical temperature stratification, and the well-mixed assumption applied in the BES can't provide the correct temperature distribution and the exhaust air temperature. Using the CFD, we could get the right exhaust air temperature and send it to the BES for the heat recovery performance calculation. Coupling BES and

CFD was also applied to predict the energy and thermal comfort performance for buildings with large space or atrium (Alnusairat et al. 2017).

Many advanced air-conditioning technologies like humidity control and zonal air-conditioning with different temperature, are used in the museums where relative humidity and temperature control is 
highlighted to conserve artworks (Ascione et al. 2013). CFD is capable to determine the moisture fluctuation in the rooms with a hygrothermal model (Steeman 2009). With these parameters the BES determines the state parameters of the HVAC equipment, like the inlet flow rate, inlet flow temperature and relative humidity (Ascione et al. 2013).

\subsubsection{Dynamics evaluation of the advanced air-conditioning control strategies}

It is difficult for BES to predict proper indoor temperature distribution in the control process for systems with nonuniform airflow distribution (Du et al. 2015). To evaluate control strategies of advanced airconditioning and ventilation systems, it is necessary to improve the airflow models adapted for control study (Jreijiry et al. 2003). The coupled simulation of CFD and BES can be utilized for simulating dynamic control strategies, such as feedback control, and Variable Air Volume (VAV) system.

Zuo, Wetter, et al. (2016) and Tian, Sevilla, Zuo, et al. (2017) coupled Fast Flow Dynamics (FFD) with HVAC models programmed in Modelica using a quasi-dynamic scheme to simulate the dynamics of different ventilation systems with feedback control. The results showed the coupled simulation could provide a more realistic environment to study the control of the stratified ventilation system. As shown in Figure 15, Modelica is used to simulate the VAV system and three zones in the space, while FFD is used to simulate the rest zone that has stratified airflow distribution due to the existing of a heated blockage. Each zone has a dedicated VAV terminal box which is control using the room-averaged temperature. With the coupled simulation, the dynamic change of the VAV system and indoor environment, such as room air temperature, supply air temperature at the terminal box, valve openings in the terminal box, pressure difference between supply and return ducts, can be obtained to improve the design and operation of the system. 


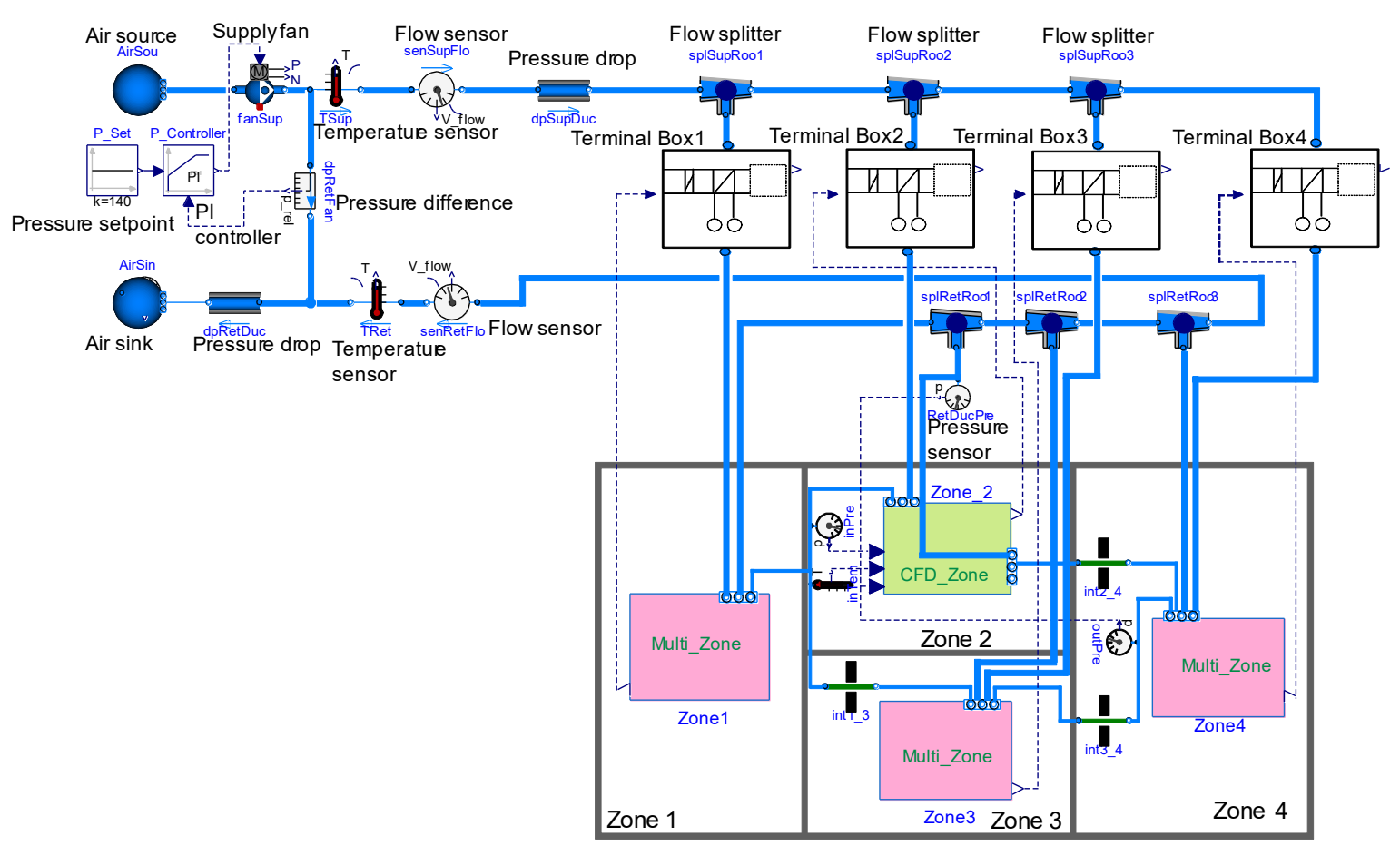

Figure 15 Sketch of VAV system connecting four zones (Tian, Sevilla, Zuo, et al. 2017).

The VAV system adjusts the supply airflow rate to meet the thermal comfort for occupants based on the temperature measured by sensors located in each thermal zone. So, the air temperature distribution and the locations of sensors have great impact on the cooling or heating performance of VAV systems. The VAV systems were studied by coupling TRNSYS and CFD (Du et al. 2015), Modelica and FFD (Tian, Sevilla, Zuo, et al. 2017) or using a CFD-based virtual test method (Sun and Wang 2010). Du et al. (2015) coupled TRNSYS and CFD with a quasi-dynamic data synchronization scheme to study the temperature sensor placement for the control of a VAV system. TRNSYS provides load, supply air temperature and flowrate to CFD. Then CFD calculates air temperature distribution, velocity distribution and return air temperature and returns the results to BES. The temperature sensor placement was optimized based on energy consumption and predicted mean vote (PMV). It was found that the temperature sensor placement may influence the supply air flow rate and air temperature and velocity distribution and then further impact on the thermal comfort of occupants and energy consumption. They also found the conventionally selected positions of the temperature sensors for the VAV terminal control can be further optimized. Sun and Wang 
(2010) used an internal coupling method and imbedded a VAV control model into CFD through user defined function (UDF) to study control of VAV system. The new application for CFD simulation can be used to evaluate control strategies for a system before it is constructed. It was found that the utilization of the virtual sensors could improve the temperature control accuracy and control reliability for the VAV system.

\section{Discussion}

Our above review and analysis has identified the potential of using coupled simulation between BES and CFD in improving design and operation of HVAC systems. Nevertheless, there exist several research gaps to broaden the application of the coupled simulation. First, CFD simulation speed should be dramatically improved to break the bottleneck of the coupled simulation speed. Second, more interfaces should be developed for CFD for easier realization of the coupled simulation. Third, reduced order models of both BES and CFD need to be developed to enable model-based control.

\subsection{Reduce Computational Cost of CFD Simulation}

CFD simulations are widely regarded as time-consuming, especially when being applied to large spaces (Zhai et al. 2002; Hosain and Fdhila 2015). There are mainly two ways to improve the simulation speed of CFD simulation. Firstly, a straightforward way is to apply CFD to the space where necessary and multizone models to the rest. Wang and Chen (2007) proposed to apply CFD to space existing non-uniform momentum and temperature distribution and multizone to the rest of the building. Based on this methodology, Tian, Sevilla, Zuo, et al. (2017) studied the control of VAV system by coupling BES, CFD, and multizone models. Surely, the fundamental way to accelerate CFD simulation is by using the advanced solving algorithms and top-of-the-line hardware. Zuo and Chen (2009) introduced FFD for airflow simulation in buildings, and using a time-splitting method, FFD can solve the Navier-Stokes equations roughly 50 times faster than CFD. More efforts have been taken to further improve the speed of FFD, such as coarse grid (Jin et al. 2015), faster solver (Jin et al. 2014), etc. Likewise, Wang et al. (2017) proposed a state-space method to accelerate CFD using linearization techniques and fast-than-real-time speed was reported. Besides developing advanced solvers, another fundamental way to expedite CFD simulation is to take 
advantage of hardware advancement by using parallel computing techniques. Wang et al. (2011) implemented CFD in OpenCL to perform parallel simulation on GPU and achieved speedup to a great extent. Zuo and Chen (2010) parallelized FFD simulation using CUDA and reported 10-30 additional speedup by running FFD on a NVIDIA GeForce 800 GTX GPU. To take advantage of heterogeneous platforms on personal computer, Tian, Sevilla, and Zuo (2017) parallelized 3 dimensional FFD simulation using OpenCL and reported over 1000 times speedup using a top-tier AMD FirePro W8100 GPU.

Zuo et al. (2014) attempted to coupled BES using Modelica models and FFD to study the dynamics of HVAC control. Even with FFD, the time cost of coupled simulation is still significantly larger than the simulation using multizone model. Thus, it remains a research topic to couple BES and CFD running on heterogeneous platforms (for example, BES on CPU and CFD on GPU) and optimize the data exchange to achieve stable and fast coupled simulation.

\subsection{Develop Interfaces for CFD Program}

As we mentioned in Section 2.4.2, the commercial and open-source CFD programs that are commonly used in simulating airflow in buildings do not provide interfaces to middleware and FMI as of October 2017. Consequently, customized interfaces are usually the only viable option to the users to couple BES and CFD. To resolve this limitation, efforts are needed to develop interfaces of CFD programs to commonly used middleware, such as BCVTB. As an open source and extensively validated CFD engine, OpenFOAM can be first used to initiate the interface development. The interface should be compatible such that it can be easily transplanted to other CFD programs. Though an intermediate agent can be developed to couple CFD with BES through middleware, middleware may have no full control on the simulators and the overhead for data exchange may increase. The second approach is to develop FMI for CFD, which seems to be a possible future trend. As discussed before, though CFX was coupled to Modelica and EnergyPlus using a customized interface (Wetter and Van Treeck 2017), no CFD programs were reported to have FMI readily available. For example, ANSYS has offered FMI capability to its system simulation program SCADE, but not yet to CFD programs, such as Fluent and CFX (Kher 2014). Although it may take substantial development efforts, having an FMI to in-house and open source CFD programs can help its integration 
with other programs and provide more functionality to its users.

\subsection{Achieve Model-based Control using Co-simulation}

Without further improving the speed of CFD simulations, model-based control using the coupled simulation is not achievable. Du et al. (2015) performed optimization study to find optimal location of thermostat to control the VAV using coupled simulation of BES and CFD. However, due to the demanding computational efforts of CFD simulations, they are required to reduce the search domain to 7 locations. Therefore, it is critical to improve the speed of CFD using reduced order model (ROM) before applying the coupled simulation in optimization study.

Zuo, Li, et al. (2016) reviewed the commonly used ROMs for CFD simulations, such as Principle Orthogonal Decomposition, Artificial Neural Network, State-Space representation, etc. However, those methods share one limitation that is when inquiry point is outside the training domain, the extrapolation may result in large prediction errors. To resolve the limitation, the authors proposed to use in situ adaptive tabulation (ISAT) which is initially developed for combustion simulation (Tian, Sevilla, Li, et al. 2017). ISAT can predetermine the accuracy of inquiry and if the prediction error exceeds a bond set by the user, it will launch a CFD simulation to answer the inquiry. In the future, it remains a research topic to harness machine learning techniques to generate ROMs with physical-based CFD program and on-site measurement.

Bin synchronization scheme can be used to coupled BES with a ROM trained by CFD simulation results (Zhai and Chen 2005). The ROM, which varies based on application, can be a database (Zhai et al. 2002), artificial network (Rong et al. 2011), or a data-fitted formula (Zhang et al. 2013; Hiyama and Kato 2011; Pan et al. 2010). Kim et al. (2015) demonstrated to use the bin synchronization scheme to couple BES with ROM of CFD for control of rooftop units. With no CFD run during the coupling, the bin synchronization scheme is computationally faster than dynamic one. Using conventional ROMs, the accuracy of the bin synchronization scheme may significantly drop if the flow conditions are outside the range of training domain (Zhai et al. 2002). Using ISAT or other self-learning ROMs, a thorough training should be performed before taking it online for real-time control, to avoid unnecessary call of CFD simulation. 
Wang and Ma (2008) reviewed optimization techniques for supervisory and optimal control for HVAC systems. However, in the context of optimization based on coupled simulation of BES and CFD, it is not clear which scheme can achieve best performance in terms of stability, efficiency, and accuracy. Moreover, when data synchronization time step is large, the outputs of the coupled simulation might not be continuous and derivative-based search cannot be used. Therefore, it remains a research topic in the future to develop guidelines and best practice rules for such optimization studies.

\subsection{Validation of Co-simulation}

Validation of co-simulation model is critical before it is adopted for design and operation applications. As our literature review identified that the co-simulation model currently targets the design application, the validation of co-simulation model was done mainly by validating the BES and CFD respectively. This is practical as on-site measurement can be impossible in the design phase. Most studies assumed the correctness and capability of the BES and CFD programs and did not provide a dedicated validation in their studies (Zhai et al. 2002). Few of the studies provided the validation of either program against experimental data (Du et al. 2015; Tian, Sevilla, Zuo, et al. 2017; Zuo, Wetter, et al. 2016) and small-scale experiments (Novoselac 2004; Wang 2007). Nevertheless, it needs future research efforts to develop small-scale experiments with high-quality measurements to validate the co-simulation on study the energy and control performance of HVAC system. Moreover, those data can also be used to study the performance of different data synchronization strategies. In the operation phase, the co-simulation model can be validated against the on-site measurements (Pan et al. 2010; Kim et al. 2015). However, in these studies, only a few scenarios can be studied due to the limitation of measurement period on site. Therefore, one interesting research topic is to perform the validation in operation phase by considering the hardware-in-the-loop using middleware interface or standard interfaces. Another interesting research topic is to automatically tune the co-simulation model using the real-time data from the building management system. 


\section{Conclusion}

With the popularity of the HVAC systems that create stratified airflow distribution in the buildings, the coupling of BES and CFD becomes an important approach to study their performances, as it showed improved performance compared to either BES or CFD standalone simulation in previous studies. This paper presented a thorough literature review on coupling BES and CFD. The focus of this paper is mainly on the external coupling, which is preferred over the internal coupling, because of the better reusability of existing BES and CFD programs and a potentially faster computation speed.

Static coupling and dynamic coupling schemes can be used to synchronize data between BES and CFD. Static coupling scheme is fast because it performs data exchange only once. However, static coupling is limited to applications of a short simulation time with relatively stable conditions. Dynamic coupling schemes that allow multiple times of data exchange is preferred for transient simulation with dynamically changing conditions. Among dynamic coupling schemes, quasi-dynamic coupling compromises between accuracy and speed, while fully dynamic coupling that requires iterations between coupled programs may achieve better accuracy with a higher computational time. The selection of the exchanged data in fully dynamic coupling may have impact on the performance of the coupled simulation in terms of stability, convergence and speed.

External coupling of BES and CFD can be implemented using customized interfaces, middleware, and standard interfaces. The customized interface allows users to determine the exchanged data and data synchronization schemes during the development process. However, besides being costly in development, the customized interface may have issues of scalability and robustness. With pre-defined interfaces and data synchronization schemes, the middleware makes implementation of coupled simulation easier and has better scalability. The standard interface arguably provides the best flexibility and functionality to the coupled simulation implementation. With the emerging of FMI, the standard interface approach has been gaining popularity. 


\section{Acknowledgment}

This research was supported by the National Science Foundation under Award No. IIS- 1633338 and the U.S. Department of Energy under Contract No. DE-EE0007688. This research was also funded in part by the Defense Threat Reduction Agency and performed under U.S. Department of Energy Contract number DE-AC02-05CH11231

\section{Reference}

Akyol, B., J. Haack, B. Carpenter, S. Ciraci, M. Vlachopoulou, and C. Tews. 2012. Volttron: An Agent Execution Platform for the Electric Power System. Proceedings of the Third international workshop on agent technologies for energy systems valencia, spain.

Akyol, B. A., J. N. Haack, B. J. Carpenter, S. Katipamula, R. G. Lutes, and G. Hernandez. 2015. "Transaction-Based Building Controls Framework, Volume 2: Platform Descriptive Model and Requirements." In.: PNNL-24395, Pacific Northwest National Laboratory, Richland, WA.

Allegrini, J., and J. Carmeliet. 2017. Simulations of Local Heat Islands in Zürich with Coupled CFD and Building Energy Models. Urban Climate. doi: https://doi.org/10.1016/i.uclim.2017.02.003.

Alnusairat, S., S. Hou, and P. Jones. 2017. Investigating Spatial Configurations of Skycourts as Buffer Zones in HighRise Office Buildings.

Andersson, C., J. Åkesson, and C. Führer. 2016. Pyfmi: A Python Package for Simulation of Coupled Dynamic Models with the Functional Mock-up Interface. Technical Report in Mathematical Sciences, 2016 (2).

Ansys, C. 2009. 12.0 User's Guide. Ansys Inc.

Ascione, F., L. Bellia, and A. Capozzoli. 2013. A Coupled Numerical Approach on Museum Air Conditioning: Energy and Fluid-Dynamic Analysis. Applied energy, 103:416-27.

Axley, J. 2007. Multizone Airflow Modeling in Buildings: History and Theory. HVAC\&R Research, 13 (6):907-28.

Beausoleil-Morrison, I. 2000. "The Adaptive Coupling of Heat and Air Flow Modelling within Dynamic Whole-Building Simulation." University of Strathclyde UK.

Blochwitz, T., M. Otter, M. Arnold, C. Bausch, C. Clauß, H. Elmqvist, A. Junghanns, J. Mauss, M. Monteiro, and T. Neidhold. 2011. The Functional Mockup Interface for Tool Independent Exchange of Simulation Models. Proceedings of the Modelica'2011 Conference, March.

Blochwitz, T., M. Otter, M. Arnold, C. Bausch, H. Elmqvist, A. Junghanns, J. Mauß, M. Monteiro, T. Neidhold, and D. Neumerkel. 2011. The Functional Mockup Interface for Tool Independent Exchange of Simulation Models. Proceedings of the Proceedings of the 8th International Modelica Conference; March 20th-22nd; Technical Univeristy; Dresden; Germany.

Bouyer, J., C. Inard, and M. Musy. 2011. Microclimatic Coupling as a Solution to Improve Building Energy Simulation in an Urban Context. Energy and Buildings, 43 (7):1549-59.

Camus, B., J. Vaubourg, Y. Presse, V. Elvinger, T. Paris, A. Tan, V. Chevrier, L. Ciarletta, and C. Bourjot. 2016. "MultiAgent Environment for Complex Systems Cosimulation (Mecsyco) - Architecture Documentation." In.

Chen, Q. 2009. Ventilation Performance Prediction for Buildings: A Method Overview and Recent Applications. Building and Environment, 44 (4):848-58.

Chen, Q., X. Peng, and A. Van Paassen. 1995. "Prediction of Room Thermal Response by CFD Technique with Conjugate Heat Transfer and Radiation Models." In.: American Society of Heating, Refrigerating and AirConditioning Engineers, Inc., Atlanta, GA (United States).

Clarke, J., W. Dempster, and C. Negrao. 1995. The Implementation of a Computational Fluid Dynamics Algorithm 
within the Esp-R System. Proc. Building Simulation'95:166-75.

Crawley, D. B., J. W. Hand, M. Kummert, and B. T. Griffith. 2008. Contrasting the Capabilities of Building Energy Performance Simulation Programs. Building and Environment, 43 (4):661-73. doi: http://dx.doi.org/10.1016/i.buildenv.2006.10.027.

Crawley, D. B., L. K. Lawrie, F. C. Winkelmann, W. F. Buhl, Y. J. Huang, C. O. Pedersen, R. K. Strand, R. J. Liesen, D. E. Fisher, and M. J. Witte. 2001. Energyplus: Creating a New-Generation Building Energy Simulation Program. Energy and Buildings, 33 (4):319-31.

Devulder, T., R. Wilson, and J. Chilton. 2007. The Thermal Behaviour of Buildings Incorporating Single Skin Tensile Membrane Structures. International Journal of Low-Carbon Technologies, 2 (2):195-213.

Djunaedy, E., J. L. Hensen, and M. Loomans. 2003. Toward External Coupling of Building Energy and Airflow Modeling Programs. ASHRAE Transactions, 109 (2):771-87.

DOE. https://energyplus.net/weather/simulation.

Dols, W. S., S. J. Emmerich, and B. J. Polidoro. 2016. Coupling the Multizone Airflow and Contaminant Transport Software Contam with Energyplus Using Co-Simulation. Proceedings of the Building simulation.

Dols, W. S., L. Wang, S. J. Emmerich, and B. J. Polidoro. 2015. Development and Application of an Updated WholeBuilding Coupled Thermal, Airflow and Contaminant Transport Simulation Program (Trnsys/Contam). Journal of Building Performance Simulation, 8 (5):326-37.

Du, Z., P. Xu, X. Jin, and Q. Liu. 2015. Temperature Sensor Placement Optimization for Vav Control Using CFD-Bes CoSimulation Strategy. Building and Environment, 85:104-13.

Fan, Y., and K. Ito. 2012. Energy Consumption Analysis Intended for Real Office Space with Energy Recovery Ventilator by Integrating Bes and CFD Approaches. Building and Environment, 52:57-67.

Foucquier, A., S. Robert, F. Suard, L. Stéphan, and A. Jay. 2013. State of the Art in Building Modelling and Energy Performances Prediction: A Review. Renewable and Sustainable Energy Reviews, 23:272-88.

Fuliotto, R., F. Cambuli, N. Mandas, N. Bacchin, G. Manara, and Q. Chen. 2010. Experimental and Numerical Analysis of Heat Transfer and Airflow on an Interactive Building Facade. Energy and Buildings, 42 (1):23-8.

Gao, N., J. Niu, and H. Zhang. 2006. Coupling CFD and Human Body Thermoregulation Model for the Assessment of Personalized Ventilation. HVAC\&R Research, 12 (3):497-518.

Haack, J., B. Akyol, C. Allwardt, S. Katipamula, Z. Beech, R. Lutes, J. Chapman, and K. Monson. 2016. Volttron ${ }^{\text {TM}: ~ U s i n g ~}$ Distributed Control and Sensing to Integrate Buildings and the Grid. Proceedings of the Internet of Things (WF-IoT), 2016 IEEE 3rd World Forum on.

Haack, J., B. Akyol, N. Tenney, B. Carpenter, R. Pratt, and T. Carroll. 2013. Volttron ${ }^{\mathrm{TM}}$ : An Agent Platform for Integrating Electric Vehicles and Smart Grid. Proceedings of the Connected Vehicles and Expo (ICCVE), 2013 International Conference on.

Han, G., J. Srebric, and E. Enache-Pommer. 2015. Different Modeling Strategies of Infiltration Rates for an Office Building to Improve Accuracy of Building Energy Simulations. Energy and Buildings, 86:288-95.

Harish, V., and A. Kumar. 2016. A Review on Modeling and Simulation of Building Energy Systems. Renewable and Sustainable Energy Reviews, 56:1272-92.

Hiyama, K., and S. Kato. 2011. Integration of Three-Dimensional CFD Results into Energy Simulations Utilizing an Advection-Diffusion Response Factor. Energy and Buildings, 43 (10):2752-9.

Hosain, M. L., and R. B. Fdhila. 2015. Literature Review of Accelerated CFD Simulation Methods Towards Online Application. Energy Procedia, 75:3307-14.

IDA-ICE. 2014. "Indoor Climate and Energy 4.1." In.

Institut für Bauklimatik, T. D. http://mastersim.sourceforge.net/.

Jin, M., W. Liu, and Q. Chen. 2014. Accelerating Fast Fluid Dynamics with a Coarse-Grid Projection Scheme. HVAC\&R Research, 20 (8):932-43.

Jin, M., W. Liu, and Q. Chen. 2015. Simulating Buoyancy-Driven Airflow in Buildings by Coarse-Grid Fast Fluid Dynamics. Building and Environment, 85:144-52.

Jreijiry, D., A. Husaunndee, C. Inard, and J. G. Villenave. 2003. Control of Ventilation in Buildings Using Simbad Building and HVAC Toolbox. Proceedings of the Proceedings of 8th International IBPSA Conference, International Building Performance Simulation Association, Eindhoven, The Netherlands.

Kher, S. "A New Model Exchange Standard - the Functional Mockup Interface." http://www.ansys- 
blog.com/functional-mockup-interface/.

Kim, D., J. Braun, E. Cliff, and J. Borggaard. 2015. Development, Validation and Application of a Coupled ReducedOrder CFD Model for Building Control Applications. Building and Environment, 93:97-111.

Li, Y., and X. Li. 2015. Natural Ventilation Potential of High-Rise Residential Buildings in Northern China Using Coupling Thermal and Airflow Simulations. Proceedings of the Building Simulation.

Liu, S., J. Liu, Q. Yang, J. Pei, D. Lai, X. Cao, J. Chao, and C. Zhou. 2014. Coupled Simulation of Natural Ventilation and Daylighting for a Residential Community Design. Energy and Buildings, 68:686-95.

Liu, W., R. Duan, C. Chen, C.-H. Lin, and Q. Chen. 2015. Inverse Design of the Thermal Environment in an Airliner Cabin by Use of the CFD-Based Adjoint Method. Energy and Buildings, 104:147-55.

Lomas, K. J. 1996. The Uk Applicability Study: An Evaluation of Thermal Simulation Programs for Passive Solar House Design. Building and Environment, 31 (3):197-206. doi: Doi 10.1016/0360-1323(95)00050-X.

Negrao, C. O. 1995. "Conflation of Computational Fluid Dynamics and Building Thermal Simulation." University of Strathclyde Glasgow.

Nielsen, P. V., and T. Tryggvason. 1998. "Computational Fluid Dynamics and Building Energy Performance Simulation." In.: Dept. of Building Technology and Structural Engineering.

Nouidui, T., M. Wetter, and W. Zuo. 2014. Functional Mock-up Unit for Co-Simulation Import in Energyplus. Journal of Building Performance Simulation, 7 (3):192-202.

Novoselac, A. 2004. Combined Airflow and Energy Simulation Program for Building Mechanical System Design.

NREL. https://github.com/NREL/EnergyPlus.

Ohba, M., and I. Lun. 2010. Overview of Natural Cross-Ventilation Studies and the Latest Simulation Design Tools Used in Building Ventilation-Related Research. Advances in Building Energy Research, 4 (1):127-66.

OpenFOAM Foundation. https://github.com/OpenFOAM/OpenFOAM-dev.

Pan, Y., Y. Li, Z. Huang, and G. Wu. 2010. Study on Simulation Methods of Atrium Building Cooling Load in Hot and Humid Regions. Energy and Buildings, 42 (10):1654-60.

Pang, X., P. Bhattacharya, Z. O'Neill, P. Haves, M. Wetter, and T. Bailey. 2011. Real-Time Building Energy Simulation Using Energyplus and the Building Controls Virtual Test Bed. Proceedings of the Proceeding of the 12th IBPSA Conference.

Pappas, A., and Z. Zhai. 2008. Numerical Investigation on Thermal Performance and Correlations of Double Skin Façade with Buoyancy-Driven Airflow. Energy and Buildings, 40 (4):466-75.

Pisello, A. L., J. E. Taylor, X. Xu, and F. Cotana. 2012. Inter-Building Effect: Simulating the Impact of a Network of Buildings on the Accuracy of Building Energy Performance Predictions. Building and Environment, 58:37-45.

RISEGrid. https://sourcesup.renater.fr/daccosim/.

Rong, Q., Y. Da, Z. Xin, and J. Yi. 2011. Research on a Dynamic Simulation Method of Atrium Thermal Environment Based on Neural Network. Building and environment.

Siebert, J., L. Ciarletta, and V. Chevrier. 2010. Agents \& Artefacts for Multiple Models Coordination: Objective and Decentralized Coordination of Simulators. Proceedings of the Proceedings of the 2010 ACM Symposium on Applied Computing.

Srebric, J., Q. Chen, and L. R. Glicksman. 2000. "A Coupled Airflow and Energy Simulation Program for Indoor Thermal Environmental Studies." In.: Massachusetts Inst. of Tech., Cambridge, MA (US).

Steeman, H.-J. 2009. "Modelling Local Hygrothermal Interaction between Airflow and Porous Materials for Building Applications." Ghent University.

Strachan, P., G. Kokogiannakis, and I. Macdonald. 2008. History and Development of Validation with the Esp-R Simulation Program. Building and Environment, 43 (4):601-9.

Sun, Z., and S. Wang. 2010. A CFD-Based Test Method for Control of Indoor Environment and Space Ventilation. Building and Environment, 45 (6):1441-7.

Tian, W., Y. Fu, Q. Wang, T. A. Sevilla, and W. Zuo. 2018. "Optimization on Thermostat Location in an Office Room Using the Coupled Simulation Platform in Modelica Buildings Library: A Pilot Study." In 2018 COBEE conference. RMIT University, Australia.

Tian, W., T. A. Sevilla, D. Li, W. Zuo, and M. Wetter. 2017. Fast and Self-Learning Indoor Airflow Simulation Based on in Situ Adaptive Tabulation. Journal of Building Performance Simulation:1-14.

Tian, W., T. A. Sevilla, and W. Zuo. 2017. A Systematic Evaluation of Accelerating Indoor Airflow Simulations Using 
Cross-Platform Parallel Computing. Journal of Building Performance Simulation, 10 (3):243-55.

Tian, W., T. A. Sevilla, W. Zuo, and M. D. Sohn. 2017. Coupling Fast Fluid Dynamics and Multizone Airflow Models in Modelica Buildings Library to Simulate the Dynamics of HVAC Systems. Building and Environment.

Tian, W., and W. Zuo. 2013. Literature Review and Research Needs to Couple Building Energy and Airflow Simulation. Proceedings of the Proceedings of the the APEC Conference on Low-carbon Towns and Physical Energy Storage.

Trcka, M., and J. Hensen. 2006. Model and Tool Requirements for Co-Simulation of Building Performance. Proceedings of the 15th IASTED, 7.

Trčka, M., J. L. Hensen, and M. Wetter. 2009. Co-Simulation of Innovative Integrated HVAC Systems in Buildings. Journal of Building Performance Simulation, 2 (3):209-30.

Trnsys, A. 2000. Transient System Simulation Program. University of Wisconsin.

Villi, G., W. Pasut, and M. De Carli. 2009. CFD Modelling and Thermal Performance Analysis of a Wooden Ventilated Roof Structure. Proceedings of the Building simulation.

Wan, M., and C. Y. Chao. 2002. Experimental Study of Thermal Comfort in an Office Environment with an Underfloor Ventilation System. Indoor and Built Environment, 11 (5):250-65.

Wang, L. 2007. "Coupling of Multizone and CFD Programs for Building Airflow and Contaminant Transport Simulations." Purdue University.

Wang, L., and Q. Chen. 2007. Validation of a Coupled Multizone-CFD Program for Building Airflow and Contaminant Transport Simulations. HVAC\&R Research, 13 (2):267-81.

Wang, L., W. S. Dols, and S. J. Emmerich. 2012. Simultaneous Solutions of Coupled Thermal Airflow Problem for Natural Ventilation in Buildings. HVAC\&R Research, 18 (1-2):264-74.

Wang, L., and N. H. Wong. 2008. Coupled Simulations for Naturally Ventilated Residential Buildings. Automation in Construction, $17(4): 386-98$.

Wang, Q., Y. Pan, M. Zhu, Z. Huang, W. Tian, W. Zuo, X. Han, and P. Xu. 2017. A State-Space Method for Real-Time Transient Simulation of Indoor Airflow. Building and Environment.

Wang, S., and Z. Ma. 2008. Supervisory and Optimal Control of Building HVAC Systems: A Review. HVAC\&R Research, $14(1): 3-32$.

Wang, Y., A. Malkawi, Y. Yi, and T. Center. 2011. Implementing CFD (Computational Fluid Dynamics) in Opencl for Building Simulation. Proceedings of the 12th international building performance simulation (building simulation 2011).

Wetter, M. 2011. Co-Simulation of Building Energy and Control Systems with the Building Controls Virtual Test Bed. Journal of Building Performance Simulation, 4 (3):185-203.

Wetter, M., and C. Van Treeck. 2017. "lea Ebc Annex 60: New Generation Computing Tools for Building and Community Energy Systems." In.

Wetter, M., W. Zuo, T. S. Nouidui, and X. Pang. 2014. Modelica Buildings Library. Journal of Building Performance Simulation, 7 (4):253-70.

Widl, E., F. Judex, K. Eder, and P. Palensky. 2015. Fmi-Based Co-Simulation of Hybrid Closed-Loop Control System Models. Proceedings of the Complex Systems Engineering (ICCSE), 2015 International Conference on.

Wittchen, K., K. Johnsen, and K. Grau. 2005. Bsim User's Guide, Danish Building Research Institute. Denmark: Hørsholm.

Yi, Y. K., and A. M. Malkawi. 2008. Site-Specific Prediction for Energy Simulation by Integrating Computational Fluid Dynamics. Proceedings of the Building Simulation.

Zeng, Z., X. Li, C. Li, and Y. Zhu. 2012. Modeling Ventilation in Naturally Ventilated Double-Skin Façade with a Venetian Blind. Building and environment.

Zhai, Z., Q. Chen, P. Haves, and J. H. Klems. 2002. On Approaches to Couple Energy Simulation and Computational Fluid Dynamics Programs. Building and Environment, 37 (8):857-64.

Zhai, Z., and Q. Y. Chen. 2003. Solution Characters of Iterative Coupling between Energy Simulation and CFD Programs. Energy and Buildings, 35 (5):493-505.

Zhai, Z. J., and Q. Y. Chen. 2005. Performance of Coupled Building Energy and CFD Simulations. Energy and Buildings, 37 (4):333-44.

Zhang, R., K. P. Lam, S.-C. Yao, and Y. Zhang. 2012a. Annual Coupled Energyplus and Computational Fluid Dynamics 
Simulation of Natural Ventilation. IBPSA-USA Journal, 5 (1):314-21.

Zhang, R., K. P. Lam, S.-C. Yao, and Y. Zhang. 2012b. Coupled Energyplus and Computational Fluid Dynamics Natural Ventilation Simulation. Proceedings of the Proceedings of 5th National SimBuild Conference.

Zhang, W., K. Hiyama, S. Kato, and Y. Ishida. 2013. Building Energy Simulation Considering Spatial Temperature Distribution for Nonuniform Indoor Environment. Building and environment.

Zhang, Y., Y. Jiang, L. Z. Zhang, Y. Deng, and Z. Jin. 2000. Analysis of Thermal Performance and Energy Savings of Membrane Based Heat Recovery Ventilator. Energy, 25 (6):515-27.

Zuo, W., and Q. Chen. 2009. Real - Time or Faster - Than - Real - Time Simulation of Airflow in Buildings. Indoor air, $19(1): 33-44$.

Zuo, W., and Q. Chen. 2010. Fast and Informative Flow Simulations in a Building by Using Fast Fluid Dynamics Model on Graphics Processing Unit. Building and Environment, 45 (3):747-57.

Zuo, W., D. Li, W. Tian, and M. Wetter. 2016. Simulation Using in Situ Adaptive Tabulation and Fast Fluid Dynamics. IBPSA-USA Journal, 6 (1).

Zuo, W., M. Wetter, D. Li, M. Jin, W. Tian, and Q. Chen. 2014. Coupled Simulation of Indoor Environment, Hvac and Control System by Using Fast Fluid Dynamics and the Modelica Buildings Library. Proceedings of the Proceedings of ASHRAE/IBPSA-USA Building Simulation Conference, Atlanta, GA, USA.

Zuo, W., M. Wetter, W. Tian, D. Li, M. Jin, and Q. Chen. 2016. Coupling Indoor Airflow, HVAC, Control and Building Envelope Heat Transfer in the Modelica Buildings Library. Journal of Building Performance Simulation, 9 (4):366-81. 